\begin{tabular}{|c|l|}
\hline Title & Characterisation of rapid al kal inisation factors in Phy scomitrium patens reveals functional conservation in tip growth \\
\hline Author(s) & Ginanjar, Eggie Febrianto; Teh, Ooi- kock; Fujita, Tomomichi \\
\hline Citation & $\begin{array}{l}\text { New Phytologist, 233(6), 2412-2457 } \\
\text { https://doi.org/L0.1111/hph.17942 }\end{array}$ \\
\hline Issue Date & $2022-03$ \\
\hline Doc URL & http://hdl.handle.net/2115/84192 \\
\hline Rights & The definitive version is available at http://onlinelibrary.wiley.com/ournal/10.1111/ISSN)1469-8137 \\
\hline Type & article \\
\hline File Information & New Phytologist Ginanjar et al.pdf \\
\hline
\end{tabular}

Instructions for use 


\title{
Characterisation of rapid alkalinisation factors in Physcomitrium patens reveals functional conservation in tip growth
}

\author{
Eggie Febrianto Ginanjar ${ }^{1 *}$ (D), Ooi-kock Teh ${ }^{2,3,4 *}$ (D) and Tomomichi Fujita ${ }^{2}$ (D) \\ ${ }^{1}$ Graduate School of Life Science, Hokkaido University, Hokkaido 060-0810, Japan; ${ }^{2}$ Faculty of Science, Hokkaido University, Hokkaido 060-0810, Japan; ${ }^{3}$ Institute for the Advancement of \\ Higher Education, Hokkaihdo University, Sapporo 060-0817, Japan; ${ }^{4}$ Present address: Institute of Plant and Microbial Biology, Academia Sinica, 128 Sec.2, Academia Rd, Nankang, Taipei, \\ Taiwan
}

\begin{abstract}
Authors for correspondence:
Ooi-kock Teh

Email: okteh@gate.sinica.edu.tw

Tomomichi Fujita

Email: tfuiita@sci.hokudai.ac.jp
\end{abstract}

Received: 15 August 2021

Accepted: 17 December 2021

New Phytologist (2022)

doi: 10.1111/nph.17942

Key words: bryophytes, Physcomitrium patens, rapid alkalinisation factors (RALFs), reverse genetics, signalling peptides, tip growth.

\section{Summary}

- Small signalling peptides are key molecules for cell-to-cell communications in plants. The cysteine-rich signalling peptide, rapid alkalinisation factors (RALFs) family are involved in diverse developmental and stress responses and have expanded considerably during land plant evolution, implying neofunctionalisations in the RALF family. However, the ancestral roles of RALFs when land plant first acquired them remain unknown.

- Here, we functionally characterised two of the three RALFs in bryophyte Physcomitrium patens using loss-of-function mutants, overexpressors, as well as fluorescent proteins tagged reporter lines.

- We showed that PpRALF1 and PpRALF2 have overlapping functions in promoting protonema tip growth and elongation, showing a homologous function as the Arabidopsis RALF1 in promoting root hair tip growth. Although both PpRALFs are secreted to the plasma membrane on which PpRALF1 symmetrically localised, PpRALF2 showed a polarised localisation at the growing tip. Notably, proteolytic cleavage of PpRALF1 is necessary for its function.

- Our data reveal a possible evolutionary origin of the RALF functions and suggest that functional divergence of RALFs is essential to drive complex morphogenesis and to facilitate other novel processes in land plants.

\section{Introduction}

An important innovation in the evolution of multicellular organisms is cell-to-cell communications that are important to coordinate development at tissue level. The importance and necessity of cell-to-cell communications are even more marked in the plant cells given the obstructive rigid cell walls. It is therefore not surprising that identities of plant cells are often determined by positional information relayed from neighbouring cells as exemplified by the cell fate specification in embryo and during root development (Nakajima etal., 2001; Friml etal., 2003; Cui et al., 2007; ten Hove etal., 2015). This mobile positional information can be macromolecules such as the classic example of SHORTROOT, a plant-specific transcription factor that is trafficked from the vasculature to the adjacent layer to regulate asymmetric divisions of the cortex endodermal initial daughter as well as endodermal specification (Helariutta etal., 2000; Nakajima etal., 2001; Cui et al., 2007); alternatively these positional cues can also be small RNA molecules such as microRNAs (miRNAs) miR165/166 that move from endodermis into the vascular cylinder to restrict PHABULOSA expression and regulate xylem differentiation (Carlsbecker et al., 2010). Signal transduction in cell-to-cell

\footnotetext{
*These authors contributed equally to this work.
}

communication is also mediated by the plasma membranelocalised receptors that form receptor-like kinase (RLK)/ligand signalling modules. The ligands that have been shown to interact with the RLKs are diverse in form and can include metabolites, cell wall polysaccharide and secreted signalling peptides (Hohmann et al., 2017; Feng et al., 2018). The most well characterised signalling peptide, CLAVATA3 (CLV3), promotes differentiation of stem cells in the shoot apical meristem upon perception by three receptor complexes and transduces signalling to restrict the expression of WUSCHEL, a transcription factor that maintains stem cell identity (Rojo et al., 2002; Müller et al., 2008; Kinoshita et al., 2010).

In plants, at least 13 peptide families have been identified and can be loosely classified based on their length, either as the small post-translationally modified peptides that are composed of 5-20 amino acids (in which the CLV3 belongs to) or as the cysteinerich polypeptides with $c$. 50 amino acids that are synthesised as precursor proteins (Tavormina et al., 2015; Breiden \& Simon, 2016). A prominent member of the latter peptide category is the rapid alkalinisation factor (RALF) that was first isolated from tobacco as peptides that could rapidly increase the medium $\mathrm{pH}$ (Pearce et al., 2001). Matrix-assisted laser desorption/ionization mass spectrometry (MALDI-MS) analysis on the high performance liquid chromatography (HPLC)-purified leaf tissues identified RALF as a $5-\mathrm{kDa}$ polypeptide that was derived from a 115 
amino acid preproprotein (Pearce etal., 2001). This founding member of the RALF peptide, RALF1, was found to arrest tomato and Arabidopsis root growth without activating the defence pathway, therefore setting it apart from other plant peptide hormones such as systemin (Murphy \& De Smet, 2014). Subsequent genome mining of other plant species shows that RALF homologues are widely represented across the plant kingdom, indicating a fundamental role in plant evolution (Sharma et al., 2016; Campbell \& Turner, 2017). Consistent with this notion, RALFs have been implicated in a wide range of processes that include cell expansion (Haruta etal., 2014), lateral root and root hair development (Wu et al., 2007; Murphy et al., 2016), pollen tube elongation/integrity (Covey et al., 2010; Ge et al., 2017) and immunity (Stegmann etal., 2017). Similar to the CLV3, RALF also transduces signalling upon binding to its cognate receptors. The most well described receptor for Arabidopsis thaliana RALF1 (AtRAFL1) is a plasma membrane-localised RLK from the Catharanthus roseus RLK1 (CrRLK1) family, termed FERONIA (FER). The AtRALF1-FER interaction was identified using a phosphoproteomic approach in which recombinant AtRALF1-treated plants showed a significant increased phosphorylated FER, as a result of FER autophosphorylation upon AtRALF1 binding (Haruta et al., 2014). To date, novel receptors and co-receptors have been determined not only for AtRALF1 but also for other RALF paralogues (Li et al., 2015; Dressano et al., 2017; Gonneau et al., 2018; Blackburn et al., 2020).

Characterisation of the AtRALF1 overexpressor and knock-out mutants strongly supports the notion that RALFs repress cell elongation and expansion in root (Mingossi et al., 2010). Although the downstream signalling output of AtRALF1-FER remains unclear, the aforementioned phosphoproteomics analysis (Haruta et al., 2014) provides clues to the potential downstream effectors. Apart from FER, abundance of phosphopeptides from three other proteins also displayed an increase upon AtRALF1 treatment and these include a plasma membrane proton pump $\mathrm{H}^{+}$-ATPase 2 (AHA2), a calcium-dependent protein kinase9 (CPK9) and a PEN3/ABCG36 transporter. It has been hypothesised that the RALF peptides seemingly affect cell and organ growth through $\mathrm{Ca}^{2+}$ signalling, mitogen activated protein (MAP) kinase signalling and $\mathrm{pH}$ modulation. As well as repressing cell expansion in root growth, the AtRALF1-FER signalling complex was also shown to promote tip growth in the root hair through phosphorylation of a translation initiation factor elF4E, thereby controlling the translational efficiency of key tip growth regulators such as ROOT HAIR DEFECTIVE 6 LIKE 4 (RSL4) and RHO GTPases in PLANTS2 (ROP2) (Zhu et al., 2020a).

The diverse roles of RALFs in various plant developmental processes is likely to be a direct consequence of the massive expansion of RALF members during land plant evolution. A comprehensive phylogeny analysis of the RALF peptides showed that RALF is absent in chlorophytes and first acquired after the evolution of embryophytes (Ghorbani et al., 2015; Campbell \& Turner, 2017). In the early diverging land plants, only one and two copies of RALF were reportedly detected in Selaginella moellendorffi and Physcomitrium patens (formerly Physcomitrella patens) respectively (Campbell \& Turner, 2017). By contrast, the number of RALFs increased significantly in angiosperms, especially in Brassicaceae (37 in Arabidopsis thaliana, 33 in Arabidopsis lyrata, 32 in Brassica rapa, 33 in Capsella rubella) through gene duplication events. The widespread presence of RALFs in early diverging lineages indicates that acquisition of RALFs was not intended for core aspects of angiosperms development processes such as pollination and root growth, as these processes are absent in the early land plants. Therefore, what is the ancestral function (s) of RALF when land plants first acquired them? To address this question, we investigated the RALF function in $P$. patens, one of the models used to study early diverging land plants. Characterisation of the RALF knock-out and overexpressor mutants indicated that RALFs have overlapping functions in promoting protonemal tip growth, showing similarities to the tip growthpromoting effects of AtRALF1 in Arabidopsis root hair (Zhu et al., 2020a). Furthermore, PpRALF2 accumulates at the edge of growing tip, indicating that it is indeed acting on tip growth.

\section{Materials and Methods}

\section{Plant materials and growth}

The moss Physcomitrium patens of Bruch \& Schimp subsp. patens was used as wild-type (WT) (Ashton \& Cove, 1977; Rensing et al., 2020). Wild-type protonematal cells and the transformants were cultivated on BCDAT agar medium under continuous white-light exposure (c. $25 \mu \mathrm{mol}$ photon $\left.\mathrm{m}^{-2} \mathrm{~s}^{-1}\right)$ at $25^{\circ} \mathrm{C}$ (Nishiyama et al., 2000). The moss tissues were homogenised in fresh sterile water every 5-7 d. Suspensions were incubated on BCDAT or BCD agar medium overlaid with the cellophane in 9-cm Petri dishes. For the inducible system, 4-d-old protonemal tissues after subculture were transferred with cellophane (Futamura Chemical, Nagoya, Japan) to agar medium containing $1 \mu \mathrm{M} \beta$-estradiol (Wako, Osaka, Japan) and used for observation subsequently.

For gametophore number scoring, protoplast-derived colonies were used. Regenerated protoplasts were transferred to a fresh BCDAT plate and incubated for 5-6 d. Established colonies were picked and grown for an additional $15-16 \mathrm{~d}$ before gametophores were scored. To induce transgene overexpression, established colonies were grown for an additional $8 \mathrm{~d}$ before being transferred to BCDAT medium supplemented with either dimethyl sulfoxide (DMSO) or $1 \mu \mathrm{M} \beta$-estradiol (Sigma) and grown for a further $7 \mathrm{~d}$ before gametophore counting by manual scoring. For subapical cell length measurement, 2-d-old protoplasts regenerated from various genotypes were transferred to BCDAT medium supplemented with $0.5 \%$ glucose (BCDATG) and cultivated for an additional $9 \mathrm{~d}$ under unilateral red light. For transgene overexpression, regenerated protoplasts were grown for $7 \mathrm{~d}$ on BCDATG medium supplemented with either DMSO (as mock) or $1 \mu \mathrm{M} \beta$-estradiol. FIJI (IMAGEJ) software was used to score subapical cell length, cell number and colony size.

To quantify the proportion of chloronemata and caulonemata in a single colony, colonies were divided to four equal parts and 20 protonemata from each part were randomly scored. The identity of the protonemata was determined by the presence of oblique or perpendicular crosswalls. 
Gene identifiers, phylogenetic tree construction and bioinformatics

Rapid alkalinisation factor amino acid sequences and respective gene identifiers were retrieved from Рнүтоzome v.13 (https:// phytozome-next.jgi.doe.gov, for species other than Arabidopsis thaliana) and TAIR v.10 (https://www.arabidopsis.org/index.jsp, for Arabidopsis thaliana) (Supporting Information Table S1). These sequences are aligned using MAFFT v.7 (https://mafft.cbrc. jp/alignment/software/) (Katoh et al., 2018) before being curated using NOISY algorithms embedded on PHYLOGENY (https:// ngphylogeny.fr) (Dress et al., 2008; Lemoine et al., 2019). The best substitution model (with the lowest Bayesian information criterion, BIC score) for these aligned and curated amino acid sequences was then predicted using the model selection tab on the IQ-TREE web server (http://iqtree.cibiv.univie.ac.at) (Trifinopoulos et al., 2016). Maximum-likelihood analysis of the amino acid sequences was then performed using the Tree Inference tab on the IQ-TREE web server by choosing the variable time (VT) substitution model and standard bootstrap analysis, with 100 bootstrap alignments.

The following web tools were used to predict the presence of signal peptides (SignalIP-5.0, http://www.cbs.dtu.dk/services/ SignalP/), S-palmitoylation (GPS-Lipid, http://lipid.biocuckoo. org/index.php) (Xie etal., 2016) and transmembrane domains (Tмнмм v.2.0, http://www.cbs.dtu.dk/services/TMHMM/). Web-based Illustrator for Biological Sequences (IBS; http:// ibs.biocuckoo.org/online.php\#) was used to draw schematic representations for genes and proteins (Liu et al., 2015).

\section{Molecular cloning and transgenic plants}

To generate knock-out mutants for PpRALFs, $c .1 .5 \mathrm{~kb}$ of the $5^{\prime}$ and $3^{\prime}$ homologous regions (HR) were PCR-amplified (primers \#468, \#469 for PpRALF1 $5^{\prime} \mathrm{HR}$; primers \#470, \#471 for PpRALF1 $3^{\prime}$ HR; primers \#472, \#473 for PpRALF2 $5^{\prime}$ HR; primers \#474, \#475 for PpRALF2 $3^{\prime}$ HR; primers \#476, \#477 for PpRALF3 $5^{\prime}$ HR; primers \#478, \#479 for PpRALF3 $3^{\prime} \mathrm{HR}$ ) from genomic DNA with PrimeSTAR Max DNA polymerase (Takara, Kusatsu, Japan). The HR fragments of PpRALF1, PpRALF2 and PpRALF3 were cloned into pTN182 (Sakakibara et al., 2008), p35S-Zeo or pTN186 vectors (Li etal., 2017), respectively, using the HotFusion method (Fu et al., 2014). To generate PpRALF1-Citrine and PpRALF2-Citrine inducible constructs, coding sequences (including $5^{\prime}$ UTR) were PCRamplified (primers \#818, \#1120 for PpRALF1; primers \#820, \#1121 for PpRALF2) using PrimeSTAR Max DNA polymerase (Takara) and cloned into pENTR1A (ThermoFisher) and sequenced, before being subcloned into pGX8Y (Kubo et al., 2013) using LR reactions to generate pGX8Y-PpRALF1 and pGX8Y-PpRALF2.

To generate in-frame knock-in PpRALF1-Citrine and PpRALF2-tagRFP constructs, $c .1 .5 \mathrm{~kb}$ of HR upstream of the stop codon (primers \#1122, \#1123 for PpRALF1; primers \#1124, \#1125 PpRALF2) and downstream of the last exon (primers \#1126, \#1127 for PpRALF1; primers \#1128, \#1129 for
PpRALF2) were PCR-amplified from genomic DNA using Tks Gflex $^{\text {TM }}$ DNA polymerase (Takara). These HR fragments from PpRALF1 and PpRALF2 were cloned into pCTRN-NPTII and pTagRFP-NPTII, respectively, using the HotFusion method (Fu et al., 2014). Tables S2 and S3 summarise the oligonucleotide sequences used in this study and recombinant plasmids generated from this study, respectively. Strategies for homologous recombination to generate knock-out mutants and knock-in reporter lines and their verifications are described in Methods S1-S6.

To generate transgenic moss, protoplasts of an appropriate genetic background were prepared and transformed with $15 \mu \mathrm{g}$ of plasmid DNA. The polyethylene glycol (PEG)-mediated transformation was performed as described previously (Nishiyama et al., 2000). The final transgenic lines were selected twice on BCDAT agar medium containing the appropriate antibiotics. Table $S 4$ summarises the transgenic lines generated and respective selection markers.

\section{RNA sequencing}

Total RNA from $16 \mathrm{~h}$-induced (either DMSO or $1 \mu \mathrm{M} \beta$ estradiol) protonemata tissues were extracted using the RNeasy Plant Mini Kit (Qiagen). RNA quality control, preparation of cDNA libraries, sequencing and raw reads processing were performed on the DNBSeq platform (BGI, Kobe, Japan) in 150-bp paired-end mode. Low-quality, adaptor-polluted and high content of unknown base reads were filtered and 42-46 million clean reds were obtained for each library. Differentially expressed genes (DEG) were identified using PossionDis algorithms.

\section{Microscopy imaging}

Confocal images were acquired using a Zeiss Laser Scanning Microscope 980. Citrine was excited using laser $514 \mathrm{~nm}$ (laser power $0.5-1 \%$ ) with emission signal collected within a 508$579 \mathrm{~nm}$ spectrum at 1 Airy Unit, in conjunction with a main dichroic beam splitter (MBS) 514/639. TagRFP was excited using a $543 \mathrm{~nm}$ laser (laser power $0.4-0.8 \%$ ) with the emission signal collected within a $543-632 \mathrm{~nm}$ spectrum at 1 Airy Unit, in conjunction with an MBS 488/561.

\section{Immunoblotting}

Protonemal tissues were ground to fine powder in liquid nitrogen followed by the addition of three volume of urea extraction buffer (4 M urea, $100 \mathrm{mM}$ dithiothreitol (DTT), 1\% Triton X-100). The homogenate was spun down for $5 \mathrm{~min}$ at $20000 \mathrm{~g}$ to remove the cell debris. The protein was mixed with protein loading buffer (10\% sodium dodecyl sulphate (SDS), $500 \mathrm{mM} \mathrm{DTT,}$ $50 \%$ glycerol, $500 \mathrm{mM}$ Tris- $\mathrm{HCl}, 0.05 \%$ bromophenol blue) and denatured at $68^{\circ} \mathrm{C}$ for $5 \mathrm{~min}$ before electrophoresis. For immunodetection of PpRALF1-Citrine and PpRALF2-Citrine, total proteins were resolved on $12 \%$ SDS-polyacrylamide gel electrophoresis (SDS-PAGE) acrylamide gels and immunoblotted with mouse monoclonal anti-green fluorescent protein (anti-GFP) JL-8 (dilution 1:1000; Takara) and anti-mouse 
immunoglobulin G (IgG) (dilution 1:5000; GE Healthcare, Hino, Japan).

\section{Results}

\section{Physcomitrium patens possesses three copies of RALFs}

To identify RALF orthologues in Physcomitrium patens (PpRALF), we queried the Physcomitrium genome (Phytozome v.13, v.3.0 assembly) using the AtRALF1 amino acid sequence. Three putative homologues were detected with $E$ values of $3.3 \mathrm{E}$ 7 (PpRALF1, gene identifier Pp3c3_15280), 3.8E-5 (PpRALF2, gene identifier Pp3c6_7200) and 2.9E-6 (PpRALF3, gene identifier Pp3c25_4180). No additional RALF candidate was detected when queries we repeated using the remaining 36 AtRALFs (Table S1). All PpRALFs are characterised by an N-terminus signal peptide and PpRALF1 possesses a canonical RxxL/RxLx site that could be subjected to SITE1-Protease (S1P) cleavage (Fig. 1a) (Matos et al., 2008; Srivastava et al., 2009). Therefore our search had uncovered an additional PpRALF as opposed to those reported previously (Campbell \& Turner, 2017). Phylogenetic analysis using selected AtRALFs of which their queries on Physcomitrium proteome returned positive hits was unable to resolve the phylogeny relationship between AtRALFs and PpRALFs (Fig. S1). We therefore sought to perform phylogenetic analyses using putative RALFs from more closely related species such as chlorophytes (marine water algae), charophytes (fresh water algae) and early land plant bryophytes. Similar to results reported previously (Campbell \& Turner, 2017), RALF homologues were missing in all nine chlorophytes queried, including Chlamydomonas reinhardtii and Volvox cateri (https://phytozomenext.jgi.doe.gov). We were also unable to identify any RALF homologues from the genomes of representative charophytes such as Mesostigma viride, Klebsormidium nitens, Chara braunii, Mesotaenium endlicherianum and Spirogloea muscicola (https:// phycocosm.jgi.doe.gov/phycocosm/home). This strongly suggested that RALF is a novel genetic invention after terrestrialisation. Indeed, putative RALFs were detected in all bryophytes: Anthoceros punctatus (a hornwort, one RALF), Marchantia polymorpha (a liverwort, three RALFs), Ceratodon purpureus (a moss, four RALFs) and Sphagnum (peat moss, four RALFs). The number of RALF homologues expanded considerably in early tracheophyte (vascular plants) such as Ceratopteris richardii (a fern, eight RALFs) and Thuja plicata (a conifer tree, 15 RALFs). Intriguingly, only 2 RALFs were found in the Selaginella moellendorff (a lycophyte) proteome.

Our phylogenetic analyses showed that PpRALF1 diverged from PpRALF2 and PpRALF3 to form two sister groups with the purple moss (Ceratodon purpureus) RALFs CepurRALFs. Notably PpRALFs seemingly shared an ancestral lineage with some of the pteridophytes and angiosperm (Arabidopsis) RALFs and formed a clade that was distinct from RALFs of other (a)
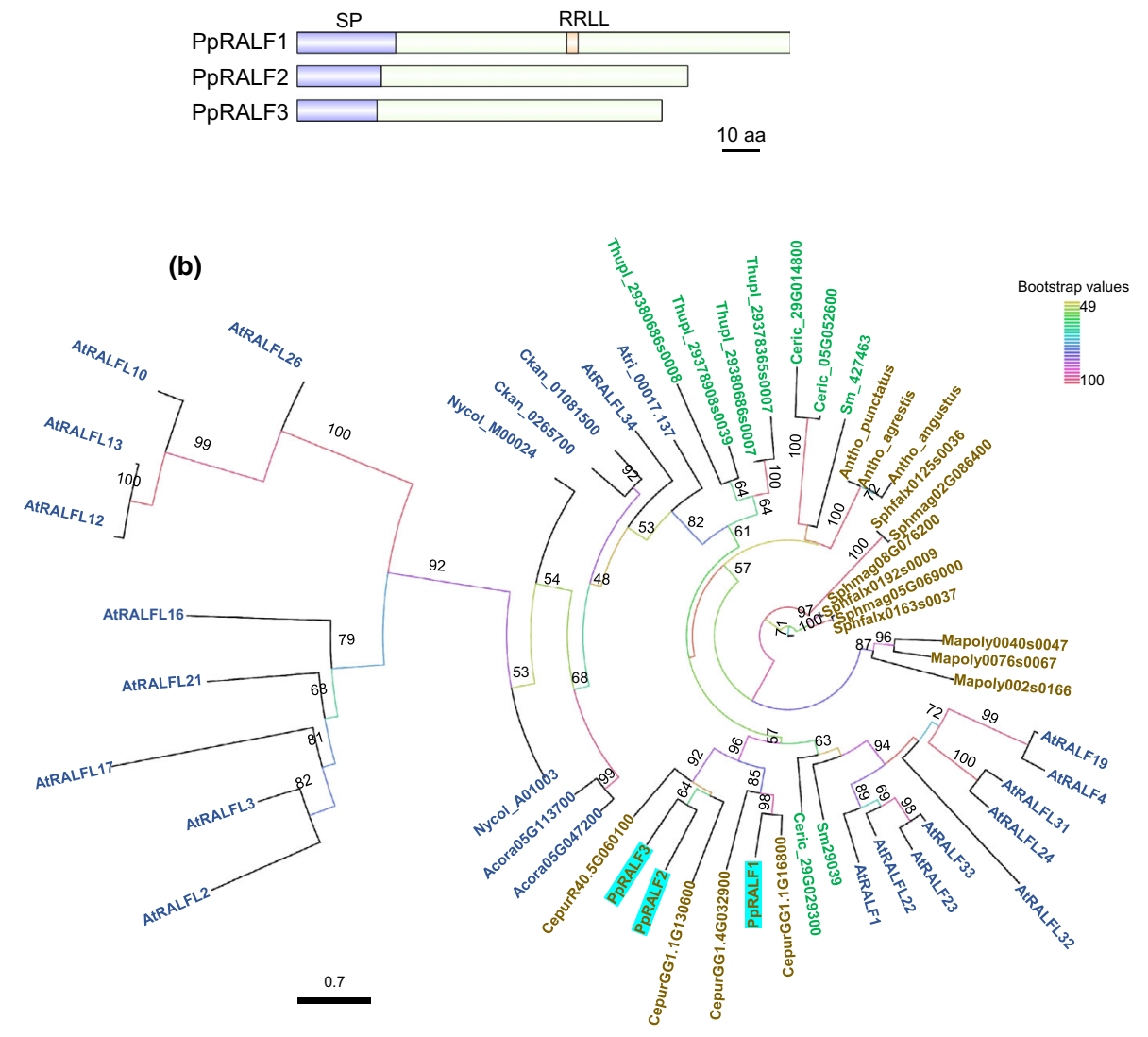

Fig. 1 Physcomitrium patens possesses three rapid alkalinisation factors (RALFs). (a) Schematic representations of the PpRALF protein domains. All RALFs are characterised by an $\mathrm{N}$-terminus signal peptide (SP; purple rectangular boxes). PpRALF1 possesses an RRLL cleavage site (red rectangular box). Bar, 10 amino acids (aa). (b) An unrooted maximum-likelihood phylogenetic tree with RALF amino acid sequences from bryophytes (brown-coloured entries), pteridophytes and gymnosperm (green-coloured entries) as well as angiosperms (blue-coloured entries). Species names are summarised as follows: Physcomitrium patens (Pp), Ceratodon purpureus (Cepur), Anthoceros agrestis (Antho_agrestis), Anthoceros punctatus (Antho_punctatus), Anthoceros angustus (Antho_angustus), Marchantia polymorpha (Mapoly), Sphagnum fallax (Sphfalx), Sphagnum magellanicum (Sphmag), Ceratopteris richardii (Ceric), Selaginella moellendorffii (Sm), Thuia plicata (Thupl), Acorus americanus (Acora), Amborella trichopoda (A.trichopoda), Nymphaea colorata (Nycol), Cinnamomum kanehirae (Ckan) and Arabidopsis thaliana (At). Numbers and colours on the node indicate bootstrap support values. Variable time (VT) substitution model with gamma distributed rates of four and invariable sites were used. Bar indicates the substitution rate. 
bryophytes such as liverwort and hornwort (Fig. 1b). Nine AtRALFs could be found within this clade and the grouping was consistent with trees published previously (Mecchia et al., 2017; Abarca et al., 2021), lending support to the reliability of our phylogenetics analyses. It is interesting to note that these AtRALFs were not functionally orthologous, with AtRALF19/4 involved in pollen tube tip growth and AtRALF23/33 required for pollen hydration (Mecchia et al., 2017; Liu et al., 2021). The remaining AtRALFs in this clade were either not functionally characterised (AtRALFL32, AtRALFL24) or have been reported to function in salt stress tolerance (AtRALFL31, AtRALFL22) (Zhao et al., 2018) and root hair tip growth/root expansion (AtRALF1) (Dressano et al., 2017; Zhu et al., 2020a). The diverse functions of AtRALF observed in this clade strongly argues for rapid neofunctionalisation and functional diversification amongst the land plant RALFs.

\section{PpRALF1 and PpRALF2 have overlapping functions in chloronemal and filament elongation}

To functionally characterise $P p R A L F \mathrm{~s}$, we generated single (Ppralf1, Ppralf2, Ppralf3), double (Ppralf1 Ppralf2, Ppralf2 Ppralf3) and triple (Ppralf1 Ppralf2 Ppralf3) knock-out mutants through a targeted homologous recombination approach to disrupt all the PpRALF loci. Given that the mutant of the AtRALFL34, ralf34, displayed unusual positioned lateral root initiations, we first sought to investigate if a similar positional defect was also observed in the Ppralf mutants. The Physcomitrium protonemata are single-layered filamentous cells from which side branches are continuously formed at the apical end of the cells (Fig. S2a). Examination of the branching pattern in all Ppralf mutants did not reveal any deviation from the usual apical-end protrusions (Fig. S2b-g), ruling out the involvement of $P p R A L F_{\mathrm{S}}$ in positional determination of side branch formation. We further examined the morphology of single-cell-derived colonies and observed an unusual smooth edge on the colonies in Ppralf1, Ppralf2, Ppralf1 Ppralf2 (from this point forwards Ppralf1,2 dko), Ppralf2 Ppralf3 (from this point forwards Ppralf2,3 dko) and Ppralf1 Ppralf2 Ppralf3 (from this point forwards Ppralf1,2,3 tko) mutants compared with the usual hairy/ rough edges seen in the WT control (Fig. 2a-n). The observed 'smooth edge' growth defects in some of the Ppralf mutants highly resembled the knock-out mutants of SUPPRESSOR OF GENE SILENCING3 (SGS3) and ROOT HAIR DEFECTIVE 6 $L I K E(R S L)$ that have been shown to exhibit defects in caulonemal formation (Menand etal., 2007b; Plavskin et al., 2016). Hairy edges observed in the WT colonies were ascribed to the long caulonemal filaments that extend from the colonies and serve as precursor cells from which leaf-like gametophores develop, as opposed to the short, chloroplast-rich chloronemal filaments (Harrison et al., 2009). We posit that the 'smooth edge' phenotype in some of the Ppralf mutants may result from reduced caulonemal filaments. Given that the 'smooth edge' phenotype is not quantifiable, we sought to obtain numerical data by measuring the number of the gametophores that should be directly proportional to the number of caulonemal filaments in each genotype. Indeed, the number of gametophores was significantly reduced in Ppralf1 $($ mean $=6.33 \pm 3.53)$, Ppralf $2($ mean $=$ $2 \pm 1.73$ ), Ppralf1,2 dko (mean $=0.78 \pm 0.92)$, Ppralf2,3 dko $($ mean $=1.88 \pm 2.20)$ and Ppralf1,2,3 tko $($ mean $=1.33 \pm 1.66)$ when compared with WT (mean $=32.06 \pm 10.67)$ and Ppralf3 $($ mean $=23.33 \pm 9.86)$, after $20 \mathrm{~d}$ of incubation on BCDAT medium (Fig. 2o). Extended incubation ( $30 \mathrm{~d})$ under the same conditions showed similar results (Fig. S3), suggesting that the phenotype could not be simply explained by delayed gametophore development in these mutants. Notably, gametophore number in Ppralf3 was comparable with that of WT (Fig. 2o), indicating that only the disruption of Ppralf1 or Ppralf 2 in the Ppralf1,3 dko and Ppralf2,3 dko was responsible for the phenotype observed. We therefore continued to functionally characterise PpRALF1 and PpRALF2. A prominent phenotypic difference between chloronema and caulonema is the perpendicular and oblique crosswalls in respective cell types. Using this as a criterion, we scored the proportion of chloronema vs caulonema cells in Ppralf1, Ppralf2 and Ppralf1,2 dko. In agreement with the reduced gametophore phenotype, the proportion of caulonemata was also drastically reduced in Ppralf1, Ppralf 2 and Ppralf1,2 dko (Fig. 2p). Therefore, the 'smooth edge' and reduced gametophore phenotypes in Ppralf1, Ppralf2 and Ppralf1,2 dko were consequences of decreased caulonemata formation.

We further determined the causative factor of reduced caulonemata formation in Ppralf1, Ppralf2 and Ppralf1,2 dko. During the early phase of haploid stage, the protonema of Physcomitrium is predominated by the chloroplast-rich chloronema cells that elongate at the rate of $c .6 \mu \mathrm{m} \mathrm{h}^{-1}$; gradually these chloronema will differentiate into caulonema cells that elongate at c. $20 \mu \mathrm{m} \mathrm{h}^{-1}$ (Menand et al., 2007a). Both chloronema and caulonema elongate by a form of tip growth (Menand etal., 2007a). The reduced caulonemal filaments in Ppralf1, Ppralf2 and Ppralf1,2 dko may indicate impaired tip growth in chloronemata that eventually affects the chloronemato-caulonema differentiation. As such, we investigated the chloronema growth under normal white-light and red-light conditions. Chloronemal growth in Ppralf1,2 dko was not visibly affected under white-light conditions; however under the redlight condition in which side branch protrusion was inhibited, the length of the chloronemal filaments in Ppralf1,2 dko was severely impaired (Fig. 2q,r). By examining subapical cells that had fully elongated, we observed a significant $c .30 \%$ reduction in Ppralf 1 (mean $=68.88 \pm 7.70 \mu \mathrm{m})$, Ppralf $2($ mean $=69.79 \pm$ $10.02 \mu \mathrm{m}$ ) and Ppralf1,2 dko cell length (mean $=66.38 \pm$ $10.14 \mu \mathrm{m}$ ) when compared with WT (mean $=96.56 \pm$ $13.17 \mu \mathrm{m})$ (Fig. 2s). As cell division is triggered when plant cells reach a defined cell size (Jones et al., 2017), reduced cell length may delay the cell cycle progression and lead to a lowered cell number. Expectedly, the cell number in chloronemal filaments was also reduced in the Ppralf1 (mean $=8.23 \pm 1.31)$, Ppralf2 $($ mean $=7.98 \pm 1.36)$ and Ppralf1,2 dko $($ mean $=6.3 \mu \mathrm{m} \pm$ 2.08) when compared with WT (mean $=16.2 \pm 2.67)$ (Fig. 2t). Consistently, the presumed delay in cell cycle progression in the Ppralf1 and Ppralf2 mutants did not affect their ploidy level (Fig. S4a). This suggests that the entire cell cycle, rather than a 


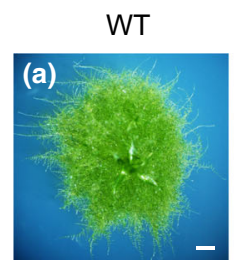

$$
\text { Ppralf1 }
$$

Ppralf2
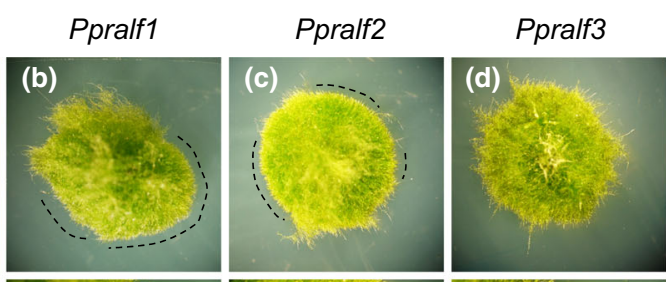

Ppralf1,2 dko
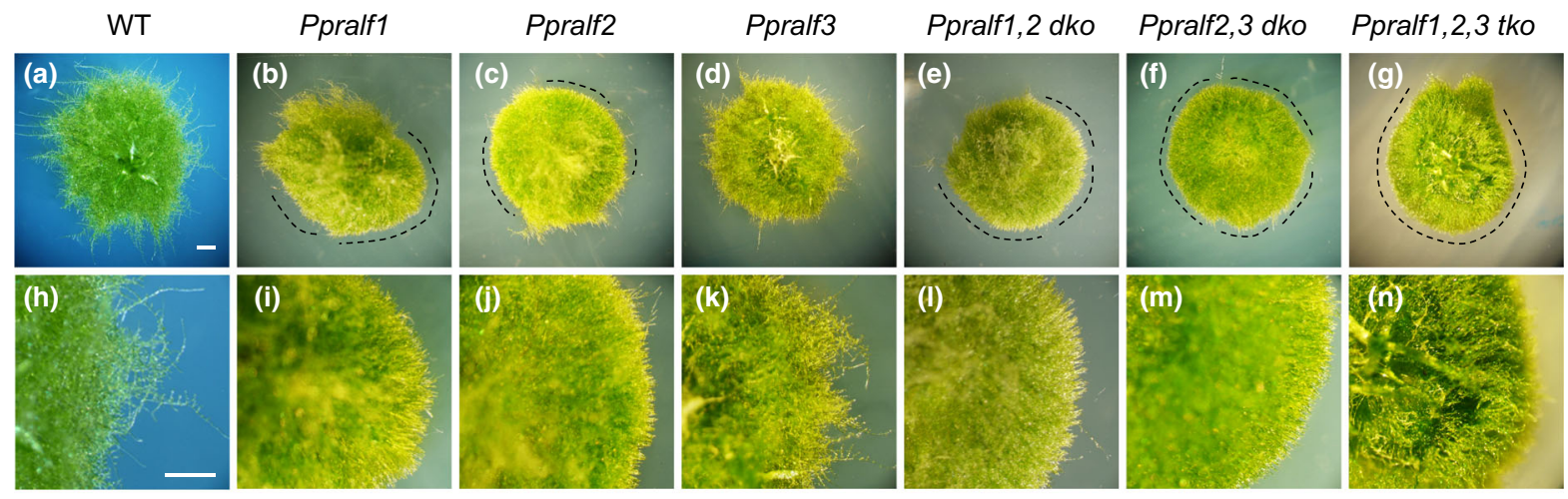

(o)
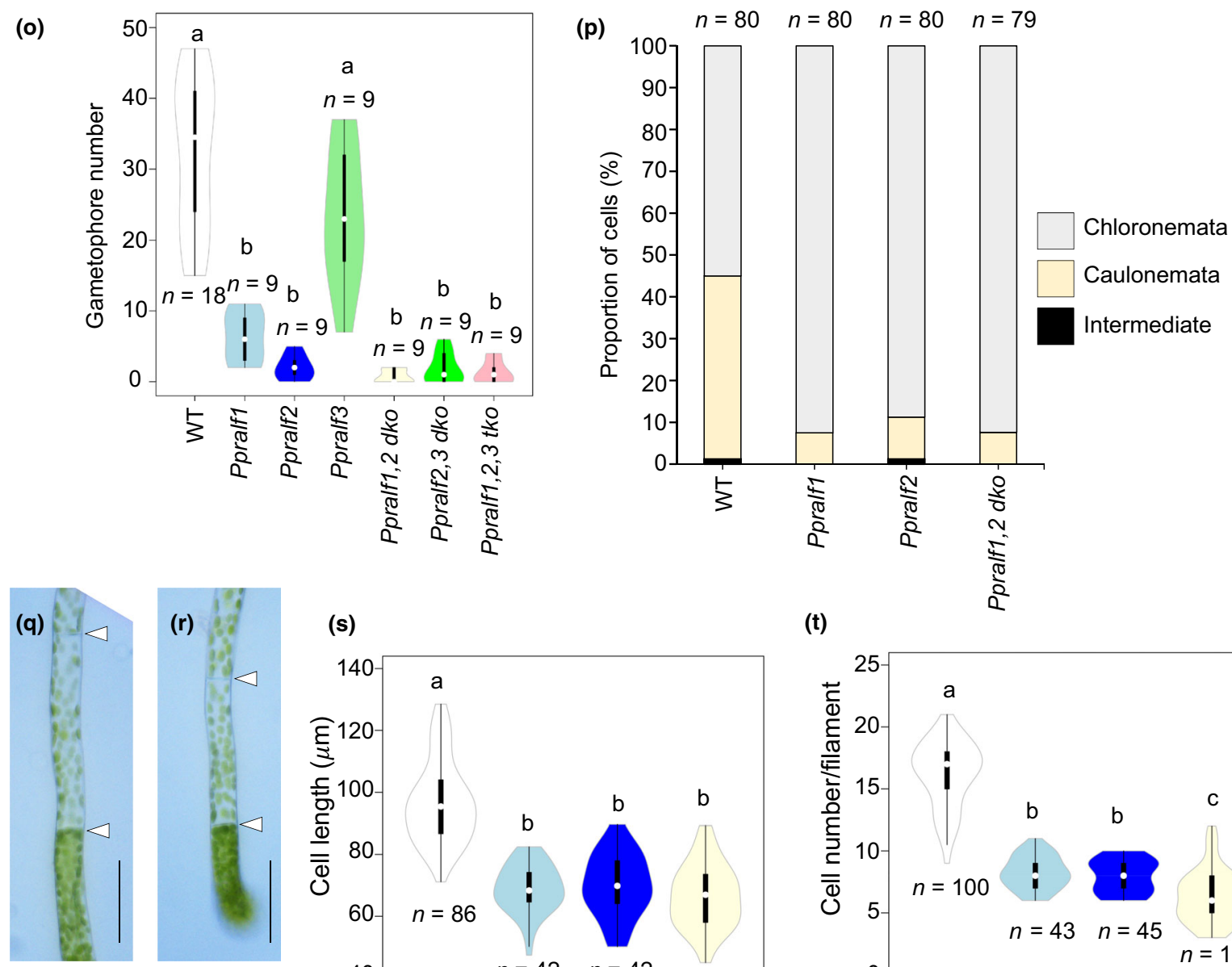

(s)

$(\mathrm{t})$
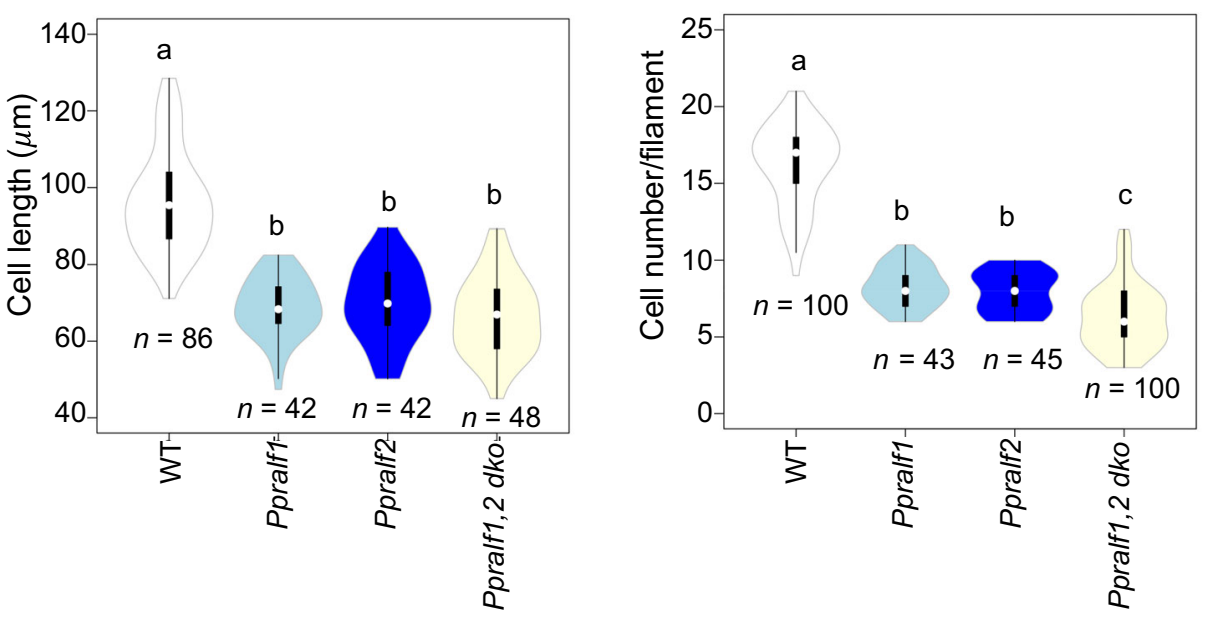

Fig. 2 PpRALF1 and PpRALF2 are involved in cell elongation. (a-n) Growth phenotype of 20-d-old colonies of wild-type (WT) (a, h), Ppralf1 (b, i), Ppralf2 $(\mathrm{c}, \mathrm{j})$, Ppralf3 (d, k), Ppralf1,2 dko (e, l), Ppralf2,3 dko (f, $\mathrm{m})$ and Ppralf1,2,3 tko (g, $\mathrm{n}$ ). Curved dashed lines indicate smooth edge. Bar, $1 \mathrm{~mm}$. (o) Violin plots depict the number of gametophore/colony in WT (white), Ppralf1 (light blue), Ppralf2 (blue), Ppralf3 (light green), Ppralf1,2 dko (light yellow), Ppralf2,3 dko (green) and Ppralf1,2,3 tko (pink). White circles show the medians; box limits indicate the $25^{\text {th }}$ and $75^{\text {th }}$ percentiles; polygons represent density estimates. Letters indicate statistically significant differences (one-way analysis of variance (ANOVA) and Tukey's post hoc test, $P<0.01$ ). $n$, sample size. (p) Stacked bars depict the proportion of chloronemal (grey), caulonemal (beige) and intermediate (black) cells in WT, Ppralf1, Ppralf2 and Ppralf1,2 dko. $n$, sample size. (q, r) Red-light-grown chloronemal filament of WT (q) and Ppralf1,2 dko (r). White arrowheads indicate crosswalls of the subapical cells. Bar, $0.5 \mathrm{~mm}$. ( $\mathrm{s}, \mathrm{t})$ Violin plots depict the subapical cell length $(\mathrm{s})$ and cell number of chloronemal filaments ( $\mathrm{t}$ ) in red-light-grown protonemata from WT (white), Ppralf1 (light blue), Ppralf2 (blue) and Ppralf1,2 dko (light yellow). White circles show the medians; box limits indicate the $25^{\text {th }}$ and $75^{\text {th }}$ percentiles; polygons represent density estimates. Letters indicate statistically significant differences (one-way ANOVA and Tukey's post hoc test, $P<0.01) . n$, sample size. 
particular cycle check-point, was slowed down. Collectively, phenotypic characterisation of the Ppralf1,2 dko showed that the primary defects of $P p R A L F 1$ and $P p R A L F 2$ disruption were in cell length and cell division, indicative of their roles in promoting the apical tip growth of the filamentous protonema.

\section{Overexpression of PpRALF1 and PpRALF2 impaired chloronemata cell length}

To substantiate the claim that PpRALF1 and PpRALF2 are involved in tip growth, we generated $\beta$-estradiol (EST) inducible overexpressor lines for PpRALF1 and PpRALF2 C-terminal Citrine-fusion reporter lines, $\mathrm{iOX}$ :PpRALF1-Citrine and $\mathrm{iOX}$ : PpRALF2-Citrine. We determined the expression levels of these transgenic lines using GFP immunoblotting and identified strong and weak expression lines for iOX:PpRALF1-Citrine and iOX: PpRALF2-Citrine (Fig. S5). Phenotypic analyses of the strongly expressed iOX:PpRALF1-Citrine (line \#1) phenocopied the Ppralf1 and Ppralf1,2 dko by exhibiting shortened chloronemata cell length (DMSO: $91.45 \pm 11.01 \mu \mathrm{m}$; EST: $57.56 \pm 9.39 \mu \mathrm{m}$, Fig. 3a,b,i) and 'smooth edge' (Fig. 3e,f), which further led to fewer caulonemata (Fig. S4b), cell numbers (DMSO: $8.89 \pm 1.14$; EST: $6 \pm 0.83$; Fig. 3 j) and gametophore numbers (DMSO: $18.4 \pm 8.26$; EST: $2.3 \pm 0.46$; Fig. $3 \mathrm{k}$ ), as well as smaller colony size (DMSO: $39.96 \pm 5.51 \mathrm{~mm}$; EST: $25.98 \pm$ $1.97 \mathrm{~mm}$; Fig. 31). Conversely, the weak iOX:PpRALF1-Citrine overexpressor (line \#2) had only a mild impact on cell length (DMSO: $81.15 \pm 10.34 \mu \mathrm{m}$; EST: $71.64 \pm 9.43 \mu \mathrm{m}$; Fig. 3c,d, i), cell numbers (DMSO: $9.06 \pm 1.46$; EST: $8.41 \pm 1.52$; Fig. 3j) and colony size (DMSO: $49.85 \pm 6.21 \mathrm{~mm}$; EST: $42.39 \mathrm{~mm} \pm 6.73 \mathrm{~mm}$, Fig. 31). The dosage-dependent cell length observed in $\mathrm{iOX}$ :PpRALF1-Citrine was indicative of the PpRALF1 function as a signalling peptide in chloronema tip growth.

When the same analyses were performed in iOX:PpRALF2Citrine strong (line \#17) and weak (line \#22) overexpressors, we observed similar phenotypes. In the strong iOX:PpRALF2Citrine overexpressor (\#17), chloronema subapical cell lengths were shortened with visibly 'smooth edge' (DMSO: $83.35 \pm$ $9.82 \mu \mathrm{m}$; EST: $54.24 \pm 8.34 \mu \mathrm{m}$; Fig. $4 \mathrm{a}, \mathrm{b}, \mathrm{e}, \mathrm{f}, \mathrm{i})$, cell numbers

Fig. 3 Overexpression of PpRALF1 impairs cell elongation. (a-d) Protonemata of strong $(a, b$, line \#1) and weak (c, d, line \#2) iOX: PpRALF1-Citrine overexpressors. Here, 7-d-old chloronemata were grown under red light on either dimethyl sulphoxide (DMSO)-supplemented (a, c) or $1 \mu \mathrm{M} \beta$-estradiol-supplemented (b, d) BCDAT medium. Arrowheads indicate cell septa. Bar, $20 \mu \mathrm{m}$. (e-h) Single-cell derived colonies (25-d-old) of strong $(e, f$, line \#1) and weak $(g, h$, line \#2) iOX:PpRALF1-Citrine overexpressors. Colonies were grown on BCDAT medium supplemented with either DMSO $(e, g)$ or $1 \mu M \beta$-estradiol $(f, h)$. Bar, $1 \mathrm{~mm}$. (i-l) Violin plots depict the subapical cell length (i) and cell number in protonemal filament (j) that were scored based on images in (a-d). Gametophore number ( $k$ ) and colony size (l) were also scored using images in (e-h). White circles show the medians; box limits indicate the $25^{\text {th }}$ and $75^{\text {th }}$ percentiles; polygons represent density estimates. One-tailed independent Student's $t$-test was used to determine significant difference. ${ }^{* *}, P<0.01$; ns, not significant. $n$, sample size.
(DMSO: $8.85 \pm 1.19$; EST: $4.98 \pm 1.07$; Fig. 4j) and colony size (DMSO: $37.62 \pm 5.76 \mathrm{~mm}$; EST: $20.98 \pm 2.98 \mathrm{~mm}$; Fig. 4l) were reduced. Similar to the weak iOX:PpRALF1-Citrine overexpressor, weak iOX:PpRALF2-Citrine (\#22) displayed only subtle

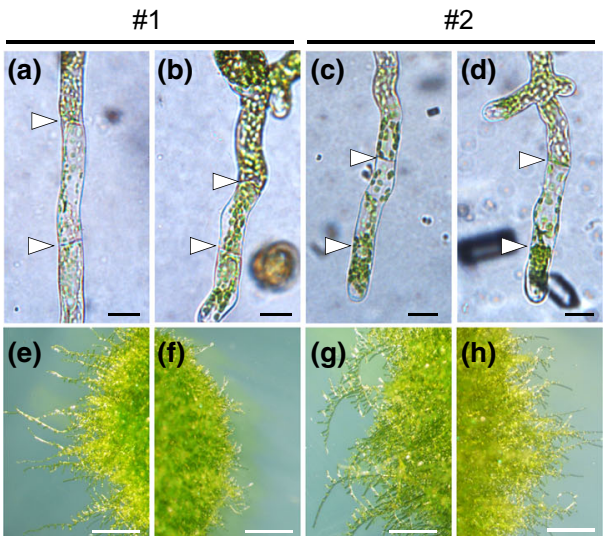

(i)

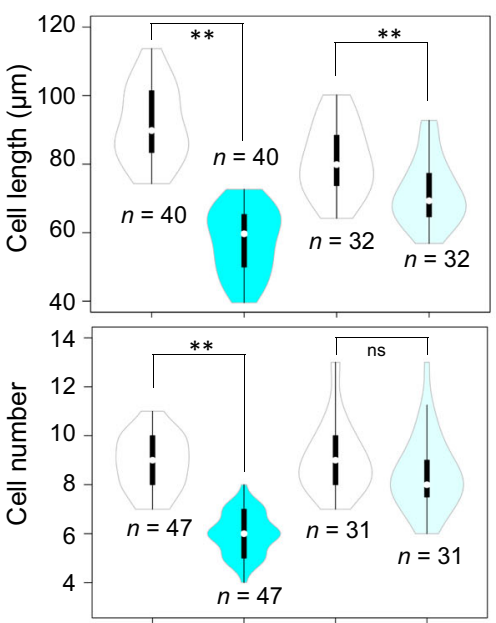

(k)

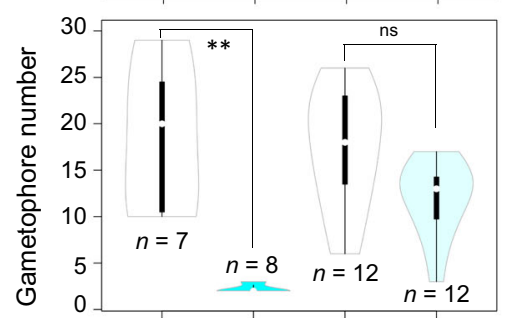

(I)

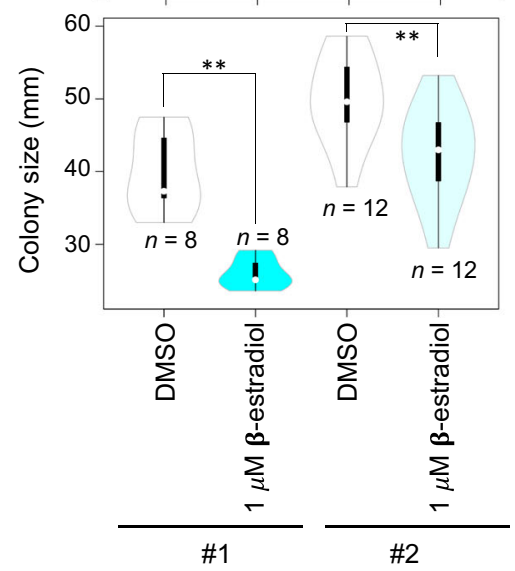


changes in chloronema subapical cell length (DMSO: $87.32 \pm$ $11.94 \mu \mathrm{m}$; EST: $77.37 \pm 11.72 \mu \mathrm{m}$; Fig. 4c,d,g,h,i), cell numbers (DMSO: $9.06 \pm 1.46$; EST: $8.42 \pm 1.52$; Fig. 4j), as well as colony size (DMSO: $49.87 \pm 5.46 \mathrm{~mm}$; EST: $46.70 \pm 5.94 \mathrm{~mm}$; Fig. 41). Notably, PpRALF2 had an additional impact on the gametophore formation as gametophore number in the weak iOX:PpRALF2-Citrine overexpressor was readily reduced to half (DMSO: $14.2 \pm 5$; EST: $7.67 \pm 3.26$; Fig. 4k) and gametophore formation was completely inhibited in the strong iOX: PpRALF2-Citrine overexpressor (DMSO: $7 \pm 2.51$; EST: 0; Fig. 4k). The PpRALF2 overexpression effect on gametophore formation was consistent with the genetic evidence in which the gametophore number in Ppralf2 $(2 \pm 1.73$; Fig. 2o $)$ was significantly reduced compared with that in Ppralf1 (6.33 \pm 3.54 ; Fig. 2o) and WT (32.06 \pm 10.67 ; Fig. 2o). Together the growth defects observed in PpRALF1 and PpRALF2 overexpressors and knock-out mutants highlighted their overlapping roles in chloronema tip growth, with PpRALF2 having an additional predominant role in gametophore formation.

\section{PpRALF1 and PpRALF2 are secreted to the plasma membrane but displayed different localisations}

A prominent characteristic of the RALF signalling peptides is the presence of an $\mathrm{N}$-terminal signal peptide for their extracellular secretion. The $\mathrm{N}$-terminus of PpRALF1 and PpRALF2 are predicted to possess a cleavable signal peptide at amino acid (aa) 126 and aa 1-23, respectively (Fig. 1a). Using the weak overexpressors of iOX:PpRALF1-Citrine and iOX:PpRALF2-Citrine that displayed no obvious growth defects upon induction (Figs 3, 4), we sought to determine the subcellular localisations of PpRALF1 and PpRALF2. In agreement with the N-terminal signal peptide, PpRALF1-Citrine and PpRALF2-Citrine were observed in the cortical endoplasmic reticulum (ER) tubular network and perinuclear membranes upon $16 \mathrm{~h}$ EST induction (Fig. S6a,b,d,e), indicating that both RALFs entered the secretory pathway. PpRALF1-Citrine and PpRALF2-Citrine are secreted to the plasma membrane but not the cell wall, as indicated by their localisation on Hechtian strands upon $0.8 \mathrm{M}$ mannitol plasmolysis (Fig. S6c,f). Notably, PpRALF2-Citrine showed

Fig. 4 Overexpression of PpRALF2 impairs cell elongation and gametophore formation. (a-d) Protonemata of strong ( $a, b$, line \#17) and weak ( $c, d$, line \#22) iOX:PpRALF2-Citrine overexpressors. Here, 7-d-old chloronemata were grown under red light on either dimethyl sulphoxide (DMSO)-supplemented $(a, c)$ or $1 \mu M \beta$-estradiol-supplemented (b, d) BCDAT medium. Arrowheads indicate cell septa. Bar, $20 \mu \mathrm{m}$. (e-h) Singlecell-derived colonies (25-d-old) of strong (e, $f$, line \#17) and weak ( $g, h$, line \#22) iOX:PpRALF2-Citrine overexpressors. Colonies were grown on BCDAT medium supplemented with either DMSO $(e, g)$ or $1 \mu M \beta-$ estradiol (f, h). Bar, $1 \mathrm{~mm}$. (i-l) Violin plots depict the subapical cell length (i) and cell number in protonemal filament (j) that were scored based on images in (a-d). Gametophore number ( $k$ ) and colony size (l) were also scored using images in (e-h). White circles show the medians; box limits indicate the $25^{\text {th }}$ and $75^{\text {th }}$ percentiles; polygons represent density estimates. One-tailed independent Student's $t$-test was used to determine significant difference. **, $P<0.01$; ns, not significant. $n$, sample size. polarised localisation at the tip of protonema (arrowhead, Fig. S6e) while PpRALF1-Citrine localised evenly on the plasma membrane (Fig. S6b), implying their different mode of action at promoting chloronema elongation. To rule out the possibility

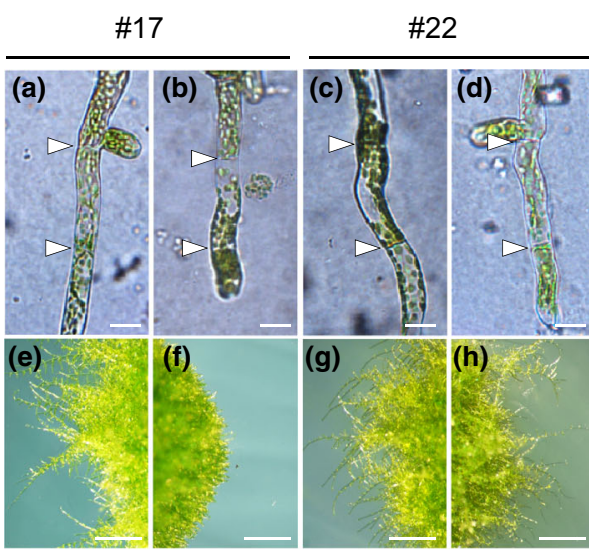

(i)

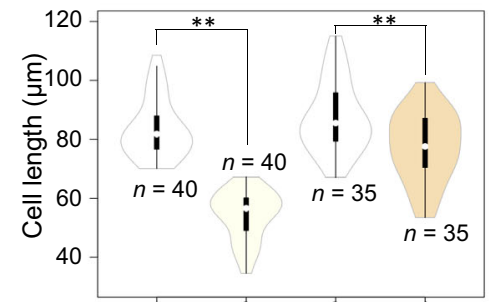

(j)

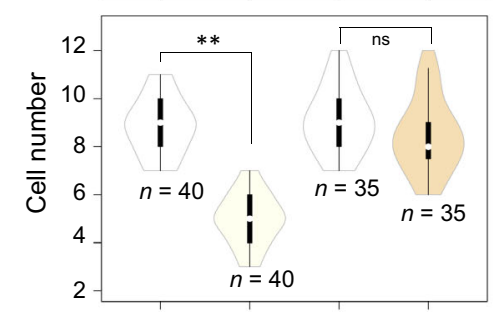

(k)

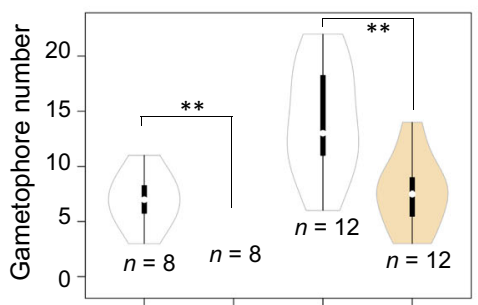

(I)

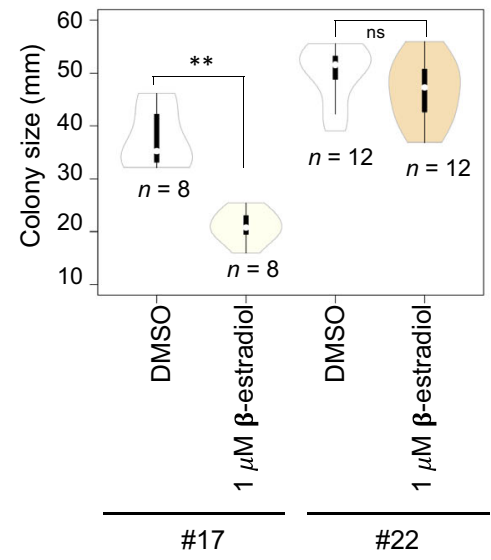


that the observed localisations of PpRALF1 and PpRALF2 were artefacts from overexpression, we used an homologous recombination approach to generate in-frame knock-in reporter lines of PpRALF1-Citrine and PpRALF2-tagRFP. When expressed under their native promoters, PpRALF1-Citrine and PpRALF2tagRFP exhibited identical subcellular localisations, as did their respective weak overexpressor lines, in which PpRALF1-Citrine was detected to localise evenly on the plasma membrane (Fig. 5a) and PpRALF2-tagRFP accumulated in a polar position at the tip of apical cells (arrowhead, Fig. 5b). Similar to the weak overexpressors, PpRALF1-Citrine and PpRALF2-tagRFP were also observed on Hechtian strands upon plasmolysis (Fig. 5c,d), indicating that they were secreted to the plasma membrane.

As PpRALF2-tagRFP displayed polarised localisation in the apical cells, we sought to understand if this localisation was correlated with the apical growth. We prepared protoplasts from PpRALF2-tagRFP-expressing moss and examined its localisation during the regeneration process. Snapshot of protoplasts from different regeneration stages showed that PpRALF2-tagRFP localised evenly on the plasma membrane before protrusion (Fig. 5e,f), but rapidly accumulated at the protrusion site when apical growth was initiated (Fig. 5g,h). The apical accumulation of PpRALF2-tagRFP persisted throughout the tip growth (Fig. 5i,j) until the first asymmetric division was completed (Fig. 5k,l). This subcellular localisation of PpRALF1 and
PpRALF2 demonstrated their involvement in facilitating protonema tip growth.

\section{Proteolytic cleavage affects PpRALF1 function}

Although both PpRALF1 and PpRALF2 are required genetically for protonema cell elongation, they are distinguishable by the presence of an S1P cleavage site in PpRALF1 (Fig. 1a). This putative cleavage site, denoted by the tetrapeptide RRLL in PpRALF1, contains a highly conserved dibasic arginine (RR) site found in yeasts and animals, and is recognised by members of the subtilisin family of serine protease (Siezen \& Leunissen, 1997). Proteolytic processing of RALFs has been shown to be important for its function in root cell expansion (Matos etal., 2008; Srivastava et al., 2009). We next asked if PpRALF1 was also proteolytically processed in vivo and how this cleavage may affect its function when replacing one of the basic amino acids for alanine (R73A; Fig. 6a).

This inducible noncleavable PpRALF1, iOX:PpRALF1 ${ }^{\text {R73A }}$ Citrine was introduced to the moss and transgenic lines with expression levels comparable with the strong iOX:PpRALF1Citrine (\#1; Fig. 3) and was chosen for further characterisations (Fig. S7). Immunoblotting of PpRALF1-Citrine, PpRALF1 ${ }^{\text {R73A }}$ Citrine and PpRALF1-Citrine showed that PpRALF1-Citrine migrated in two forms, the unprocessed proPpRALF1 (predicted MW $40.3 \mathrm{kDa}$ ) and the predominant mature cleaved
Fig. 5 PpRALF1 and PpRALF2 displayed different subcellular localisations during apical growth. (a-d) Here, 5-d-old protonemata of PpRAFL1-Citrine $(a, c)$ and PpRALF2-tagRFP $(b, d)$ incubated on either BCDAT medium $(a, b)$ or plasmolysed with $0.8 \mathrm{M}$ mannitol (c, d). Confocal images shown are z-stack projections (sum), magenta indicates chloroplasts and green indicates Citrine $(a, c)$. TagRFP is pseudocoloured as green $(b, d)$ for easier visualisation. Arrowheads indicate apical accumulation of PpRALF2-tagRFP. Bar, $10 \mu \mathrm{m}$. (e-l) Confocal images of regenerating protoplasts from PpRALF2-tagRFP.

Fluorescence (e, $g, i, k)$ and bright field $(f, h$, j, l) images shown are z-stack projections (sum). Magenta indicates chloroplasts and green indicates tagRFP. Arrowheads indicate apical accumulation of PpRALF2-tagRFP. Bar, $10 \mu \mathrm{m}$
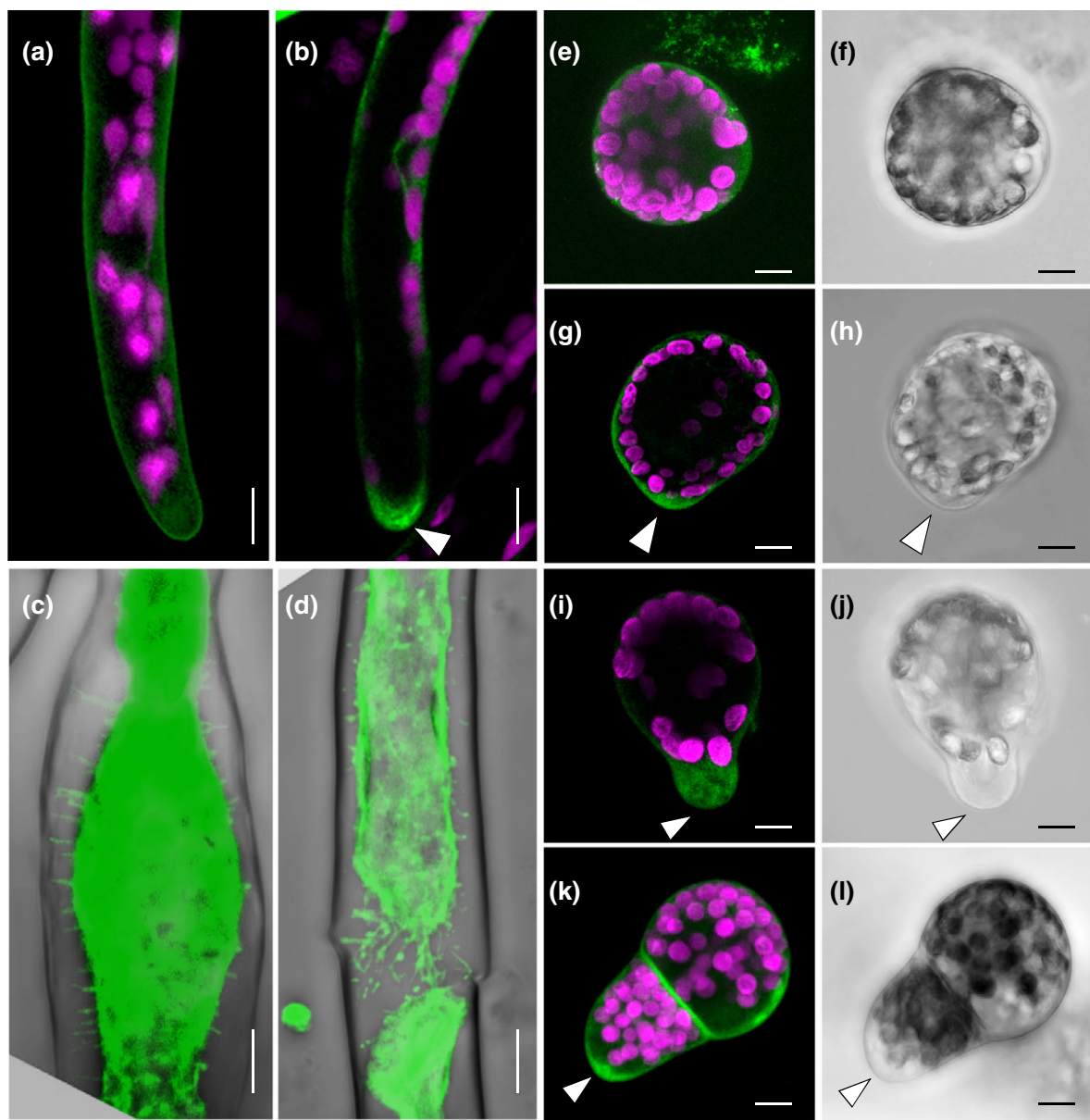
(a)
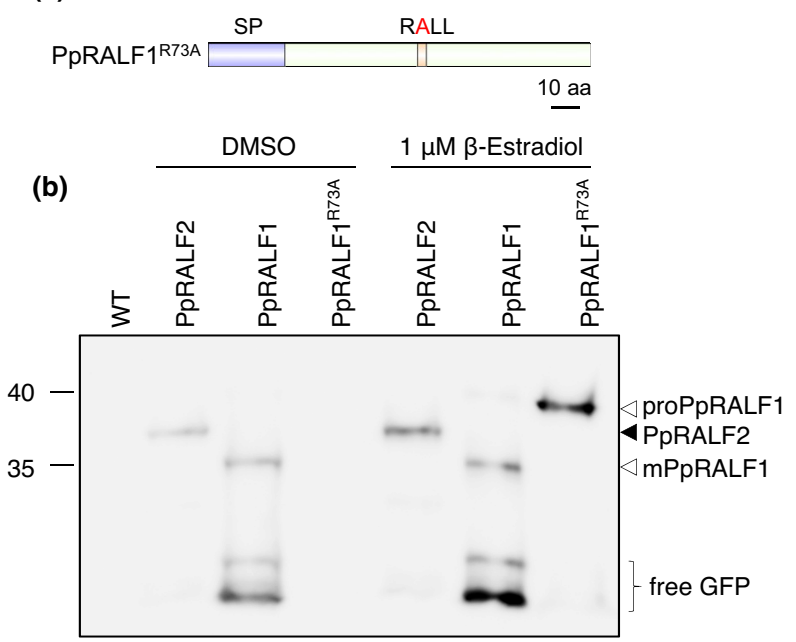

(c)

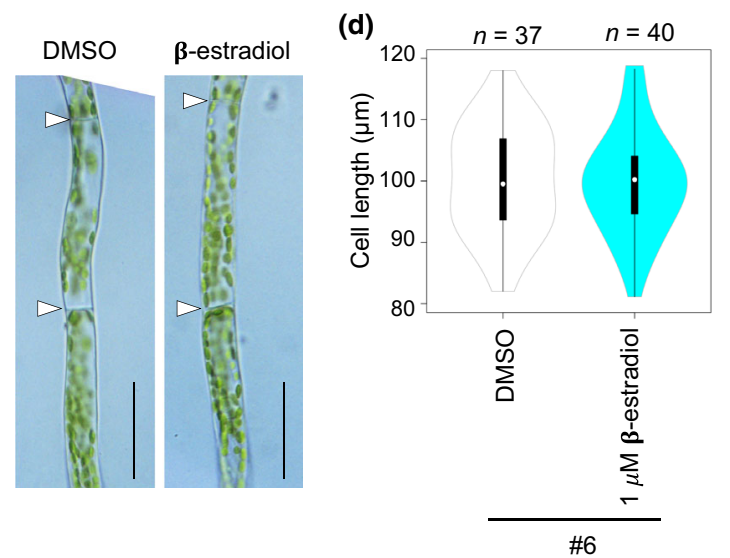

Fig. 6 Proteolytic processing of PpRALF1 is necessary for its function. (a) A schematic representation of PpRALF1 ${ }^{R 73 A}$. The R73A mutation is indicated by a red-coloured A. Bar, 10 aa (b) Green fluorescent protein (GFP) immunoblot of RALF1-Citrine, RALF1 ${ }^{\text {R73A }}$-Citrine and RALF2-Citrine. Numbers on the left indicate molecular weight of ladder in kDa. Whitefilled arrowheads on the right indicate propeptide PpRALF1 (proPpRALF1) and mature PpRALF1 (mPpRALF1) while black-filled arrowhead indicates PpRALF2. (c) Representative images of 7-d-old protonemata from iOX: RALF1 ${ }^{\text {R73A }}$-Citrine (line \#6). Red-light-grown chloronemata were incubated on dimethyl sulphoxide (DMSO)-supplemented or $1 \mu \mathrm{M} \beta$ estradiol-supplemented BCDAT medium. Arrowheads indicate cell septa. Bar, $0.5 \mathrm{~mm}$. (d) Violin plots depicts the subapical cell length of DMSOtreated or $1 \mu \mathrm{M} \beta$-estradiol-treated iOX: RALF1 ${ }^{\text {R73A }}$-Citrine (line \#6). White circles show the medians; box limits indicate the $25^{\text {th }}$ and $75^{\text {th }}$ percentiles; polygons represent density estimates. $n$, sample size.

mPpRALF1 (predicted MW $35.3 \mathrm{kDa}$ ) that migrated faster than PpRALF2-Citrine (predicted MW $37.6 \mathrm{kDa}$ ) (Fig. 6b). Conversely, PpRALF1 ${ }^{\mathrm{R} 73 \mathrm{~A}}$-Citrine migrated as an uncleaved form (Fig. 6b), suggesting that the monobasic site is no longer efficiently processed by proteases. We next characterised morphologically the iOX:PpRALF $1^{\mathrm{R} 73 \mathrm{~A}}$-Citrine (\#6) line by measuring the subapical cell length in chloronemata and found no significant difference upon PpRALF ${ }^{\mathrm{R} 73 \mathrm{~A}}$ expression (DMSO: 100.29 \pm $8.75 \mu \mathrm{m}$; EST: $99.77 \pm 8.50 \mu \mathrm{m}$; Fig. $6 c, d)$. Collectively we concluded that PpRALF1 was indeed proteolytically cleaved in vivo at the S1P cleavage site and that this was necessary for the PpRALF1 function.
PpRALF1 and PpRALF2 regulate distinct but overlapping genes to mediate tip growth

As the genetic evidence indicated that PpRALF1 and PpRALF2 function overlappingly to promote tip growth, we sought to perform RNA sequencing (RNAseq) to gain insights into the gene regulatory network controlled by PpRALF1 and PpRALF2 when their cognate receptors were activated. To this end we performed RNAseq using 5-d-old protonemata from iOX:PpRALF1-Citrine (\#1) and iOX:PpRALF2-Citrine (\#17) after $16 \mathrm{~h}$ of $\beta$-estradiol induction, a time point when the tip growth inhibition began to be observed. We detected more than 9000 differentially expressed genes (DEGs) $\left(-1 \leq \log _{2}\right.$ fold change $\left.\geq 1\right)$ when either PpRALF1 or PpRALF2 was overexpressed, of which 3178 DEGs were commonly regulated by both RALFs (Fig. 7a). By filtering for DEGs that showed a fold change of $-2 \leq \log _{2}$ fold change $\geq 2$, in total 1948 commonly regulated DEGs were further identified. Clustering analysis of these DEGs showed that PpRALF1 and PpRALF2 indeed regulated the expression patterns of two gene clusters (clusters 1 and 4) in a similar manner (Fig. 7b) while the remaining two gene clusters (clusters 2 and 3) showed opposite regulation (Fig. 7b). The overlapping functions between PpRALF1 and PpRALF2 were also reflected in gene ontology (GO) enrichment analysis (Tables S5, S6). GO terms related to cellular compartments such as nucleus (GO: 0005634), intracellular and membrane bound organelles (GO: 0005622, 0043227, 0043231, 0043229, 0043226) were commonly enriched (Fig. 7c,d; Tables S5, S6). Notably Cellular Compartments of 'kinesin complex' (GO: 0005871) and 'microtubule associated complex' (GO: 0005875) that facilitated tip growth in $P$. patens were also enriched (Hiwatashi et al., 2014; Yamada \& Goshima, 2018), supporting our claim that PpRALF1 and PpRALF2 are involved in tip growth.

While a range of biological process GO terms in biosynthesis and metabolic turnover processes were enriched in both PpRALFs, we detected distinct Molecular Function GO terms between PpRALF1 and PpRALF2. For example, phosphatidylinositol phosphate (PIP) $5^{\prime}$ dephosphorylation (GO: 0052866, 0004439, 0034593, 0034595, 0106019) was specifically overrepresented in the iOX:PpRALF1, whereas signalling receptorrelated molecular functions (GO: 0038023, 0019199, 0004888, 0060089) were only enriched in the iOX:PpRALF2 (Fig. 7c,d; Tables S5, S6). These enriched GO terms were consistent with the physiological changes such as rapid secretion and maintenance of polarised membrane domains that take place during the polarised tip growth. In conclusion, transcriptomics changes observed in the PpRALF overexpressors strongly supported the notion that PpRALF1 and PpRALF2 cooperatively mediated overlapping functions that are necessary to promote tip growth.

\section{Discussion}

\section{Functional conservation of PpRALFs and AtRALF1 in tip growth}

During terrestrialisation, land plants have acquired complex three-dimensional (3D) body plans to cope with the new 
(a)

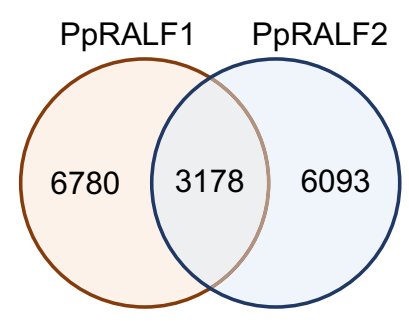

(b)

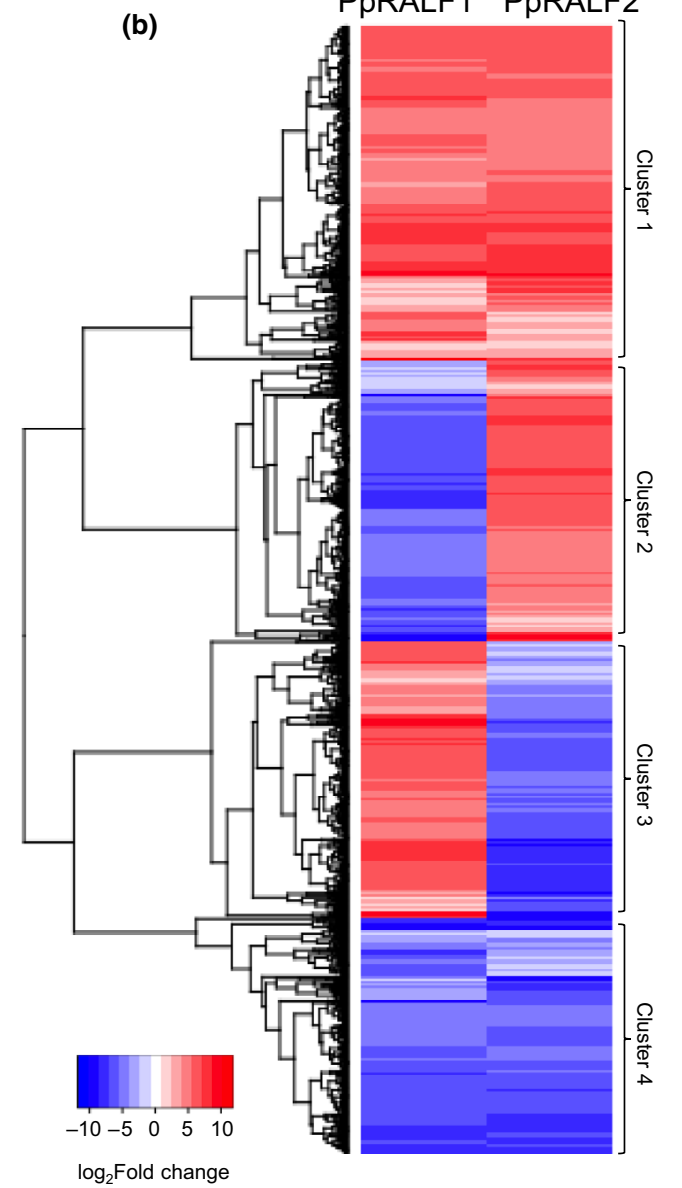

(c)

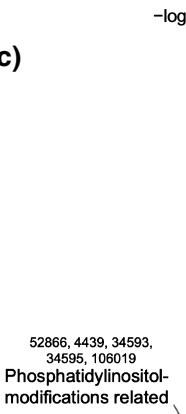

$\log (P$-values $)$

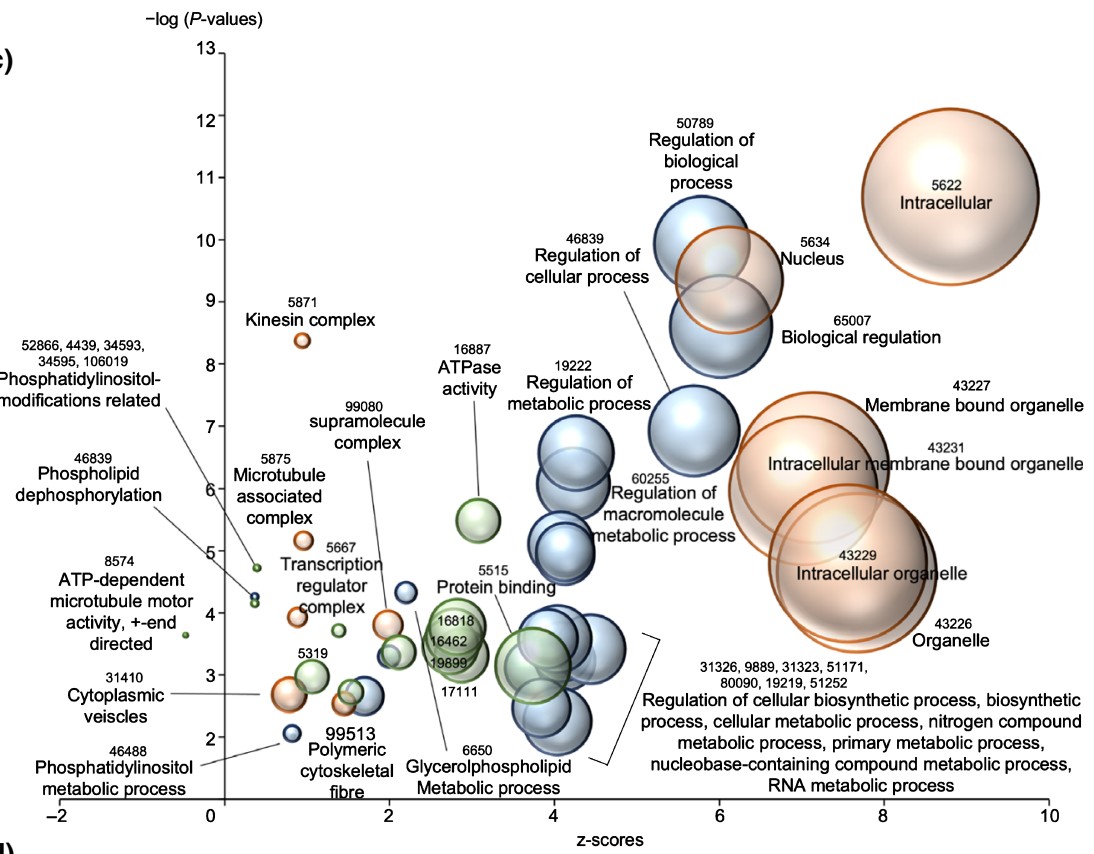

(d)

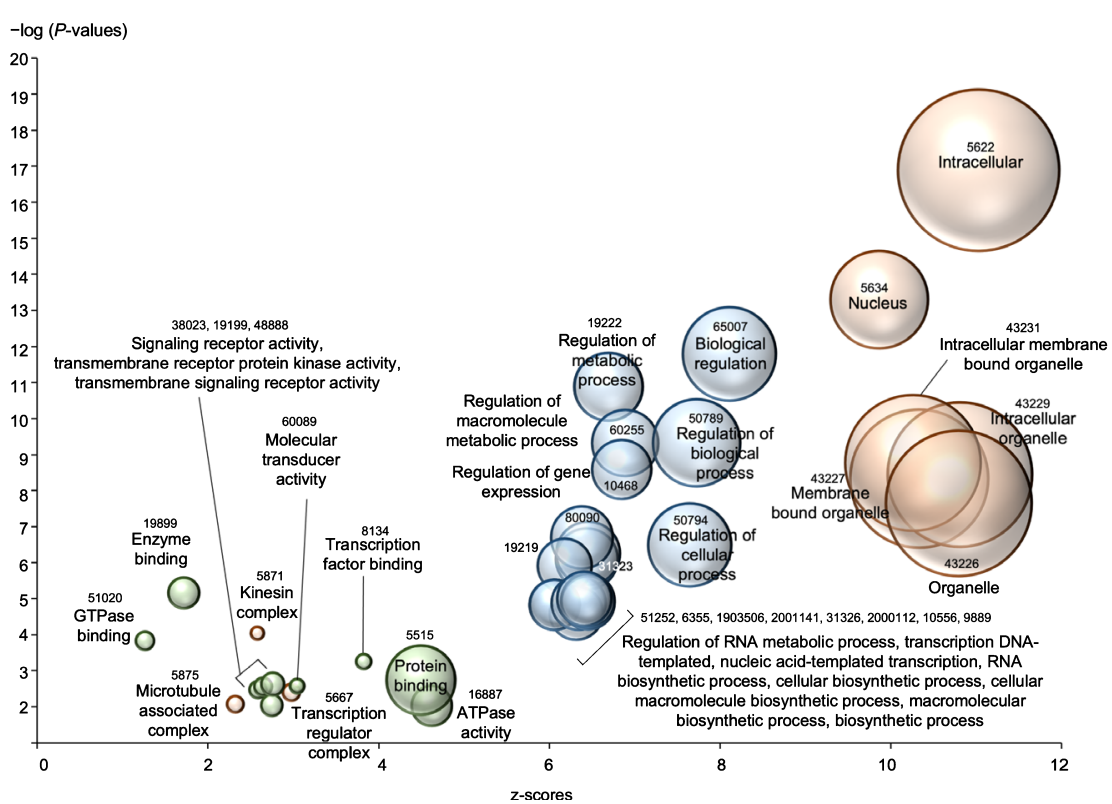

Fig. 7 Overlapped and distinct transcriptional regulation patterns of PpRALF1 and PpRALF2. (a) Venn diagram that summarises the number of differentially expressed genes (DEGs) $\left(-1 \leq \log _{2}\right.$ fold change $\geq 1$ ) between PpRALF1 (beige-coloured circle) and PpRALF2 (blue-coloured circle). (b) Heatmap that depicts the clustering analysis of overlapped DEGs $\left(-2 \leq \log _{2}\right.$ fold change $\left.\geq 2\right)$ between PpRALF1 and PpRALF2. (c) A bubble plot that depicts enriched GO terms (false discovery rate (FDR) adjusted $P$-values $\leq 0.05$ ) that are related to biological processes (blue), cellular compartments (beige) and molecular functions (green) after $16 \mathrm{~h}$ of PpRALF1 overexpression. Terms are shown as -log (adj $P$-value) ( $y$-axis) and $z$-scores ( $x$-axis) that predict the bias in gene regulation. Size of the bubbles is proportional to the number of DEGs. Numbers next to bubbles are Gene Ontology (GO) identifiers. (d) A bubble plot that depicts enriched GO terms (FDR adjusted $P$-values $\leq 0.05$ ) that are related to biological processes (blue), cellular compartments (beige) and molecular functions (green) after $16 \mathrm{~h}$ of PpRALF2 overexpression. Terms are shown as -log (adj $P$-value) ( $y$-axis) and z-scores $(x$-axis) that predict the bias in gene regulation. Size of the bubbles is proportional to the number of DEGs. Numbers next to bubbles are GO identifiers.

challenges on dry land. Two mechanisms, namely the tip growthmediated branching and rotation of the cell division plane, are thought to contribute to the two-dimensional (2D) and eventually 3D morphological complexity (Harrison, 2017; Buschmann, 2020). Genetics and morphological analyses on root hair-like rhizoids in earlier land plant models such as mosses (Physcomitrium patens) and liverwort (Marchantia polymorpha) have uncovered genetic novelties used by early land plants to mediate tip growth (Vidali etal., 2010; Jang \& Dolan, 2011; Wu etal., 2011; Honkanen et al., 2016). Some of these genetic tools are ancient and highly conserved, for example a group of basic helix-loophelix (bHLH) transcription factors ROOT HAIR DEFECTIVE 
6 (RHD6) functions in promoting the rhizoids and root hair development of bryophytes and angiosperms, respectively (Menand et al., 2007b; Honkanen \& Dolan, 2016). Conversely, the small signalling peptide CLV3 was co-opted to regulate the orientation of cell division plane through a RECEPTOR-LIKE PROTEIN KINASE 2 (RPK2) signalling pathway that is shared between Physcomitrium and Arabidopsis (Whitewoods etal., 2018) and thereby contributes to the $2 \mathrm{D}$-to-3D transitionary growth in land plants. This finding highlights the multifaceted roles played by signalling peptides in plant growth and development (Czyzewicz et al., 2013). In line with their diversified functions, the angiosperm Arabidopsis genome encodes a staggering over 1000 putative small signalling peptide sequences (Lease $\&$ Walker, 2006), making functional characterisation a challenging task. It is clear that the highly elaborated signalling peptide repertoire has resulted from multiple gene duplication events, as is the case for RALFs (Campbell \& Turner, 2017). While it is of great importance to delineate the signalling mechanisms elicited by these signalling peptides, an equally important question is their evolutionary origin. We approached this question by examining the PpRALFs in modern mosses Physcomitrium patens, which is often used as a model to understand novel morphological complexities acquired by early diverging land plants (Vidali \& Bezanilla, 2012; de Keijzer et al., 2021).

Here, we report that the PpRALFs diverged from RALFs in liverworts and tracheophytes and formed a specific sister clade that is distinct from other RALFs (Fig. 1b), leading to the speculation that PpRALFs may not be functionally orthologous to RALFs in tracheophytes and in liverworts. Unexpectedly, we found that PpRALF1 and PpRALF2 were involved in promoting protonema tip growth (Fig. 2), a function that is similar to the positive regulation of AtRALF1 in root hair tip growth (Zhu et al., 2020a,b). Upon activation by AtRALF1, the FER phosphorylates eIF4EI, a translation initiation factor that enhances mRNA binding ability with RSL4 to increase its protein synthesis rate (Zhu et al., 2020a). Although root systems are thought to have arisen independently in tracheophytes (Hetherington \& Dolan, 2018), root hairs seem to share some morphological/functional similarities with the protonemata/rhizoids of bryophytes in which both are tip-growing filamentous cells that are important for nutrient uptake and better anchorage to substrate (soil) (Jones $\&$ Dolan, 2012). Strikingly, both root hairs and rhizoid development is dependent on a bHLH transcription factor RSL of which the function has been conserved for $>420$ million years (Myr) since mosses and vascular plants diverged (Menand et al., 2007b). Our findings demonstrate the analogous function between PpRALF1/2 and AtRALF1 in tip growth promotion and provide an additional feature similarity in the tip growth mechanism between bryophytes and vascular plants. This may indicate a functional conservation of RALF after $P$. patens diverged from tracheophytes and angiosperms. It will be necessary to perform cross-species complementation of RALF to determine whether PpRALF $1 / 2$ and AtRALF 1 are functionally orthologous.

Conversely, RALF is also known to inhibit root cell expansion in angiosperms such as Arabidopsis (Matos et al., 2008; Srivastava et al., 2009), tobacco (Wu et al., 2007), sugarcane (Mingossi et al., 2010) and dandelion (Wieghaus etal., 2019). In many cases the inhibitory effect was demonstrated by the application of synthetic RALF peptides that suppressed root growth (Abarca et al., 2021). Markedly, exogenous treatment of synthetic AtRALF1 peptide also enhanced root hair length (Zhu etal., 2020a), pointing to the fact that RALF exerts tissue-specific functions in a modular manner by binding to different combinations of receptors and co-receptors. Notably, the inhibitory effects of angiosperm RALFs on root growth is also shared by a variety of fungal phytopathogens such as Fusarium oxysporum (Masachis et al., 2016; Thynne et al., 2017), leading to the speculation that fungal phytopathogens may acquire RALFs from their host plants through horizontal gene transfer and exploit RALFs to enhance infectious potential and suppress host immunity.

\section{Overlapping functions of PpRALF1 and PpRALF2 in protonemal tip growth}

Our genetic data revealed the overlapping functions of PpRALF1 and PpRALF2 in promoting chloronemal tip growth. There are two scenarios that set out how this can be achieved at the molecular level. On the one hand, PpRALF1 and PpRALF2 might form a heterodimer before receptor binding and induce downstream signalling that is necessary to promote tip growth. Heterodimerisation between signalling peptides, although rare, has recently been reported for the nonsecreted cysteine-rich peptides (NCRPs). These NCRPs, named the cysteine-rich transmembrane module (CYSTM) are described by 13 loci in the Arabidopsis genome (Xu etal., 2018) and can function as homodimers or heterodimers to regulate salt stress (Xu et al., 2018). On the other hand, it is equally possible that PpRALF1 and PpRALF2 individually bind to different RLKs on the cell surface to recruit additional co-receptors for signalling event initiation, as has been reported for RALF-mediated pollen tube elongation (Ge etal., 2019). During the tip growth of pollen tube through the female pistil tissues, pollen tube integrity is maintained by the interaction between autocrine AtRALF4 and AtRALF19 and the surface-localised receptor complex ANXUR1/2 (ANX1/2) and Buddha's Paper Seal1/2 (BUPS1/2) (Miyazaki et al., 2009; Ge etal., 2017). The presence of the AtRALF4/AtRALF19 further enhances the heterodimerisation between ANX/BUPS and their co-receptors, LORELEI-likeglycosylphosphatidylinositol-anchored proteins (LLG) (Ge et al., 2019). Indeed, structural analysis of the RALF23-induced FERLLG1 heterocomplex suggests that RALF can serve as a molecular glue between receptors and co-receptors, thereby increasing its diversity in regulating multiple processes (Xiao etal., 2019). Identifying the binding receptors for PpRALF1 and PpRALF2 will be instrumental in delineating the signalling mechanisms in tip growth promotion.

It is intriguing to note that the iOX:RALF1-Citrine and iOX: RALF2-Citrine overexpressors phenocopied the loss-of-function alleles by exhibiting shortened protonemata. Although overexpressors are expected to display enhanced tip growth as exogenous treatment of synthetic AtRALF1 did to root hair tip growth (Zhu et al., 2020a), it is not unusual that hypermorphic 
and loss-of-function alleles exhibit a similar phenotype (Gonneau et al., 2018). A possible explanation for this phenomenon is that PpRALF overexpression imposes a titration effect that dilutes out the receptor activation responses, therefore rendered the tip growth dysfunctional.

\section{Polarised localisation of PpRALF2 in the control of protonemal tip growth}

It is interesting to note that although both PpRALF1 and PpRALF2 are secreted to the plasma membrane (Fig. 5c,d), they displayed rather different subcellular localisations, with PpRALF1 symmetrically localised on the plasma membrane, while PpRALF2 accumulating at the growing tip (Fig. 5a,b). The symmetrical localisation of PpRALF1 is likely to correspond to the cleaved mPpRALF1 that represents the predominant form (Fig. 6b). The apical localisation of PpRALF2 is also shared by AtRALF1 that promotes tip growth (Zhu et al., 2020a) and AtRALF4/AtRALF19 that regulates pollen tube tip growth (Ge et al., 2017). Polarised protein localisation is common in eukaryotes and plays crucial roles in tissue patterning during plant morphogenesis (Qi \& Greb, 2017; Yang et al., 2020). One of the most prominent polarity proteins is Rho of Plants (ROP), plant-specific small GTPases that are similar to the Rho/RAC/CDC42 in other eukaryotes, which are master regulators for cell polarity in plants (Fu \& Yang, 2001). In P.patens, ROP4 is localised at the growing tip of chloronemata and at the incipient sites of side branches, presumably to activate effectors that are necessary for cell wall remodelling before and during tip growth (Ito etal., 2014; Cheng et al., 2020; Yi \& Goshima, 2020) The PpRALF1 symmetrical localisation on plasma membrane and apical accumulation of PpRALF2 at the growing tip provide a clue to the cooperative roles of PpRALF1/2, in which PpRALF1 may activate cell wall-localised receptors that facilitate cell wall remodelling, while PpRALF2 initiates signalling pathways that are crucial for tip growth, possibly through a mechanism that is analogous to that of AtRALF1 (Zhu et al., 2020a).

Unlike STOMAGEN and CLV3 that are secreted to the apoplast (Rojo et al., 2002; Sugano et al., 2010), we found that PpRALF1 and PpRALF2 are both localised on the plasma membrane, as indicated by their association with the Hechtian strands after plasmolysis. This observation is consistent with that of AtRALF1 (Campos et al., 2018). Membrane association of PpRALF2 can be explained by a putative transmembrane domain at aa 44 to aa 61 (Fig. S8). However, PpRALF1 possesses no transmembrane domain, therefore it is likely that its anchorage to the plasma membrane is facilitated by lipidation events such as $S$ palmitoylation. Indeed, we detected several putative $S$ palmitoylation sites in both PpRALF1 (aa 27 and aa 128; Fig. S8) and PpRALF2 (aa 101; Fig. S8). Furthermore, the plasma membrane localisation of PpRALF1, PpRALF2 and AtRALF1 may reflect their autocrine signalling nature, as opposed to the paracrine signalling nature of STOMAGEN and CLV3 that affects neighbouring cells (Schoof et al., 2000; Wang et al., 2021).

\section{Conclusions}

Gene duplication and functional diversification is an important driving force for evolutionary innovation and organismal adaptation (Conant \& Wolfe, 2008). Our results provide a possible evolutionary route for land plant RALFs and suggest that the ancestral function of RALFs is likely to promote tip growth, an important morphogenesis feature acquired by the land plant after bryophytes diverged from the sister lineage of charophytes. During land plant evolution, RALFs have undergone multiple events of tandem duplication (Cao \& Shi, 2012; Campbell \& Turner, 2017) and functional differentiation that eventually led to neofunctionalisation of RALFs in the tracheophytes and served to communicate signals in various developmental, reproduction and immunity processes.

\section{Acknowledgements}

We thank Professor Mitsuyasu Hasebe (NIBB, Japan) for sharing pTN182, pTN186, p35SZeo, pCTRN-NPTII and pTagRFPNPTII. This work was funded by the NOVARTIS Foundation (Japan) for the Promotion of Science (grant ID 3133, to O-KT) and MEXT/JSPS KAKENHI Grant no. JP19K06701 (O-KT), JP16K14747, JP18H04829 and JP20H04878 (TF). EFG is supported by a Japanese government MEXT scholarship. The authors declare no conflict of interest.

\section{Author contributions}

TF and O-KT conceptualised and designed the research. O-KT and EFG performed experiments and analysed data. O-KT wrote the manuscript with input from all the authors. All authors were involved in the manuscript revision. EFG and O-KT contributed equally to this work.

\section{ORCID}

Tomomichi Fujita (D) https://orcid.org/0000-0003-1434-8705 Eggie Febrianto Ginanjar (D) https://orcid.org/0000-0003-35600367

Ooi-kock Teh (iD https://orcid.org/0000-0001-6800-2748

\section{Data availability}

The data that support the findings of this study are available in the Supporting Information of this article.

\section{References}

Abarca A, Franck CM, Zipfel C. 2021. Family-wide evaluation of RAPID ALKALINIZATION FACTOR peptides. Plant Physiology 187: 996-1010. Ashton NW, Cove DJ. 1977. The isolation and preliminary characterisation of auxotrophic and analogue resistant mutants of the moss, Physcomitrella patens. Molecular and General Genetics 154: 87-95.

Blackburn MR, Haruta M, Moura DS. 2020. Twenty years of progress in physiological and biochemical investigation of RALF peptides. Plant Physiology 182: $1657-1666$. 
Breiden M, Simon R. 2016. Q\&A: how does peptide signaling direct plant development? BMC Biology 14: 58.

Buschmann H. 2020. Into another dimension: how streptophyte algae gained morphological complexity. Journal of Experimental Botany 71: 3279-3286.

Campbell L, Turner SR. 2017. A comprehensive analysis of RALF Proteins in green plants suggests there are two distinct functional groups. Frontiers in Plant Science 8: 37.

Campos WF, Dressano K, Ceciliato PHO, Carlos Guerrero-Abad J, Leonir Silva A, Fiori CS, Do Canto A, Bergonci T, Claus L, Silva-Filho M et al. 2018. Arabidopsis thaliana rapid alkalinization factor 1-mediated root growth inhibition is dependent on calmodulin-like protein 38. Journal of Biological Chemistry 293: 2159-2171.

Cao J, Shi F. 2012. Evolution of the RALF gene family in plants: gene duplication and selection patterns. Evolutionary Bioinformatics 2012: $271-$ 292.

Carlsbecker A, Lee JY, Roberts CJ, Dettmer J, Lehesranta S, Zhou J, Lindgren O, Moreno-Risueno M, Vatén A, Thitamadee $S$ et al. 2010. Cell signalling by microRNA165/6 directs gene dose-dependent root cell fate. Nature 465: 316321.

Cheng X, Mwaura BW, Chang Stauffer SR, Bezanilla M. 2020. A fully functional ROP fluorescent fusion protein reveals roles for this GTPase in subcellular and tissue-level patterning. Plant Cell 32: 3436-3451.

Conant GC, Wolfe KH. 2008. Turning a hobby into a job: how duplicated genes find new functions. Nature Reviews Genetics 9: 938-950.

Covey PA, Subbaiah CC, Parsons RL, Pearce G, Lay FT, Anderson MA, Ryan C, Bedinger P. 2010. A pollen-specific RALF from tomato that regulates pollen tube elongation. Plant Physiology 153: 703-715.

Cui H, Levesque MP, Vernoux T, Jung JW, Paquette AJ, Gallagher KL, Wang JY, Blilou I, Scheres B et al. 2007. An evolutionarily conserved mechanism delimiting SHR movement defines a single layer of endodermis in plants. Science 316: 421-425.

Czyzewicz N, Yue K, Beeckman T, De Smet I. 2013. Message in a bottle: small signalling peptide outputs during growth and development. Journal of Experimental Botany 64: 5281-5296.

Dress AWM, Flamm C, Fritzsch G, Grünewald S, Kruspe M, Prohaska SJ, Stadler P. 2008. Noisy: identification of problematic columns in multiple sequence alignments. Algorithms for Molecular Biology 3: 7.

Dressano K, Ceciliato PHO, Silva AL, Guerrero-Abad JC, Bergonci T, OrtizMorea FA et al. 2017. BAK1 is involved in AtRALF1-induced inhibition of root cell expansion. PLoS Genetics 13: e1007053.

Feng W, Kita D, Peaucelle A, Cartwright HN, Doan V, Duan Q, Liu M, Maman J, Steinhorst L, Schmitz-Thom I et al. 2018. The FERONIA receptor kinase maintains cell-wall integrity during salt stress through $\mathrm{Ca}^{2+}$ signaling. Current Biology 28: 666-675.

Friml J, Vieten A, Sauer M, Weijers D, Schwarz H, Hamann T, Offringa R, Jürgens G. 2003. Efflux-dependent auxin gradients establish the apical-basal axis of Arabidopsis. Nature 426: 147-153.

Fu C, Donovan WP, Shikapwashya-Hasser O, Ye X, Cole RH. 2014. Hot fusion: an efficient method to clone multiple DNA fragments as well as inverted repeats without ligase. PLoS ONE 9: e115318.

Fu Y, Yang Z. 2001. Rop GTPase: a master switch of cell polarity development in plants. Trends in Plant Science 6: 545-547.

Ge Z, Bergonci T, Zhao Y, Zou Y, Du S, Liu MC, Luo X, Ruan H, García-Valencia L, Zhong S et al. 2017. Arabidopsis pollen tube integrity and sperm release are regulated by RALF-mediated signaling. Science 358: $1596-1600$.

Ge Z, Zhao Y, Liu MC, Zhou LZ, Wang L, Zhong S, Hou S, Jiang J, Liu T, Huang $Q$ et al. 2019. LLG2/3 are co-receptors in BUPS/ANX-RALF signaling to regulate Arabidopsis pollen tube integrity. Current Biology 29: 3256-3265.

Ghorbani S, Lin YC, Parizot B, Fernandez A, Njo MF, Van De Peer Y, Beeckman T, Hilson P. 2015. Expanding the repertoire of secretory peptides controlling root development with comparative genome analysis and functional assays. Journal of Experimental Botany 66: 5257-5269.

Gonneau M, Desprez T, Martin M, Doblas VG, Bacete L, Miart F, Sormani R, Hématy K, Renou J, Landrein B et al. 2018. Receptor kinase THESEUS1 is a rapid alkalinization factor 34 receptor in Arabidopsis. Current Biology 28 : 2452-2458.
Harrison CJ. 2017. Development and genetics in the evolution of land plant body plans. Philosophical Transactions of the Royal Society B: Biological Sciences 372: 20150490.

Harrison CJ, Roeder AHK, Meyerowitz EM, Langdale JA. 2009. Local cues and asymmetric cell divisions underpin body plan transitions in the moss Physcomitrella patens. Current Biology 19: 461-471.

Haruta M, Sabat G, Stecker K, Minkoff BB, Sussman MR. 2014. A peptide hormone and its receptor protein kinase regulate plant cell expansion. Science 343: 408-411.

Helariutta Y, Fukaki H, Wysocka-Diller J, Nakajima K, Jung J, Sena G et al. 2000. The SHORT-ROOT gene controls radial patterning of the Arabidopsis root through radial signaling. Cell 101: 555-567.

Hetherington AJ, Dolan L. 2018. Stepwise and independent origins of roots among land plants. Nature 561: 235-238.

Hiwatashi Y, Sato Y, Doonan JH. 2014. Kinesins have a dual function in organizing microtubules during both tip growth and cytokinesis in Physcomitrella patens. Plant Cell 26: 1256-1266.

Hohmann U, Lau K, Hothorn M. 2017. The structural basis of ligand perception and signal activation by receptor kinases. Annual Review of Plant Biology 68: 109-137.

Honkanen S, Dolan L. 2016. Growth regulation in tip-growing cells that develop on the epidermis. Current Opinion in Plant Biology 34: 77-83.

Honkanen S, Jones VAS, Morieri G, Champion C, Hetherington AJ, Kelly S, Proust H, Saint-Marcoux D, Prescott H, Dolan L. 2016. The mechanism forming the cell surface of tip-growing tooting cells is conserved among land plants. Current Biology 26: 3238-3244.

ten Hove CA, Lu K-J, Weijers D. 2015. Building a plant: cell fate specification in the early Arabidopsis embryo. Development 142: 420-430.

Ito K, Ren J, Fujita T. 2014. Conserved function of Rho-related Rop/RAC GTPase signaling in regulation of cell polarity in Physcomitrella patens. Gene 544: $241-247$

Jang G, Dolan L. 2011. Auxin promotes the transition from chloronema to caulonema in moss protonema by positively regulating PpRSL1and PpRSL2 in Physcomitrella patens. New Phytologist 192: 319-327.

Jones AR, Forero-Vargas M, Withers SP, Smith RS, Traas J, Dewitte W, Murray J. 2017. Cell-size dependent progression of the cell cycle creates homeostasis and flexibility of plant cell size. Nature Communications 8: 1-13.

Jones VAS, Dolan L. 2012. The evolution of root hairs and rhizoids. Annals of Botany 110: 205-212.

Katoh K, Rozewicki J, Yamada KD. 2018. MAFFT online service: multiple sequence alignment, interactive sequence choice and visualization. Briefings in Bioinformatics 20: 1160-1166.

de Keijzer J, Rios AF, Willemsen V. 2021. Physcomitrium patens: a single model to study oriented cell divisions in $1 \mathrm{D}$ to $3 \mathrm{D}$ patterning. International Journal of Molecular Sciences 22: 2626.

Kinoshita A, Betsuyaku S, Osakabe Y, Mizuno S, Nagawa S, Stahl Y, Simon R, Yamaguchi-Shinozaki K, Fukuda H, Sawa S. 2010. RPK2 is an essential receptor-like kinase that transmits the CLV3 signal in Arabidopsis. Development 137: 3911-3920.

Kubo M, Imai A, Nishiyama T, Ishikawa M, Sato Y, Kurata T, Hiwatashi Y, Reski R, Hasebe M. 2013. System for stable $\beta$-estradiolinducible gene expression in the moss Physcomitrella patens. PLoS ONE 8: e77356.

Lease KA, Walker JC. 2006. The Arabidopsis unannotated secreted peptide database, a resource for plant peptidomics. Plant Physiology 142 831-838.

Lemoine F, Correia D, Lefort V, Doppelt-Azeroual O, Mareuil F, CohenBoulakia S, Gascuel O. 2019. NGPhylogeny.fr: new generation phylogenetic services for non-specialists. Nucleic Acids Research 47: W260-W265.

Li C, Sako Y, Imai A, Nishiyama T, Thompson K, Kubo M, Hiwatashi Y, Kabeya Y, Karlson D, Wu SH et al. 2017. A Lin28 homologue reprograms differentiated cells to stem cells in the moss Physcomitrella patens. Nature Communications 8: 14242.

Li C, Yeh FL, Cheung AY, Duan Q, Kita D, Liu MC, Maman J, Luu E, Wu B, Gates L et al. 2015. Glycosylphosphatidylinositol-anchored proteins as chaperones and co-receptors for FERONIA receptor kinase signaling in Arabidopsis. eLife 4: e06587. 
Liu C, Shen L, Xiao Y, Vyshedsky D, Peng C, Sun X, Liu Z, Cheng L, Zhang $\mathrm{H}, \mathrm{Han} \mathrm{Z}$ et al. 2021. Pollen PCP-B peptides unlock a stigma peptide-receptor kinase gating mechanism for pollination. Science 372: 171-175.

Liu W, Xie Y, Ma J, Luo X, Nie P, Zuo Z, Lahrmann U, Zhao Q, Zheng Y, Zhao Y et al. 2015. IBS: an illustrator for the presentation and visualization of biological sequences. Bioinformatics 31: 3359-3361.

Masachis S, Segorbe D, Turrà D, Leon-Ruiz M, Fürst U, El Ghalid M, Leonard G, López-Berges MS, Richards TA, Felix G et al. 2016. A fungal pathogen secretes plant alkalinizing peptides to increase infection. Nature Microbiology 1 : 16043.

Matos JL, Fiori CS, Silva-Filho MC, Moura DS. 2008. A conserved dibasic site is essential for correct processing of the peptide hormone AtRALF1 in Arabidopsis thaliana. FEBS Letters 582: 3343-3347.

Mecchia MA, Santos-Fernandez G, Duss NN, Somoza SC, Boisson-Dernier A, Gagliardini V, Martínez-Bernardini A, Fabrice T, Ringli C, Muschietti J et al. 2017. RALF4/19 peptides interact with LRX proteins to control pollen tube growth in Arabidopsis. Science 358: 1600-1603.

Menand B, Calder G, Dolan L. 2007a. Both chloronemal and caulonemal cells expand by tip growth in the moss Physcomitrella patens. Journal of Experimental Botany 58: 1843-1849.

Menand B, Yi K, Jouannic S, Hoffmann L, Ryan E, Linstead P, Schaefer D, Dolan L. 2007b. An Ancient mechanism controls the development of cells with a rooting function in land plants. Science 316: 1477-1480.

Mingossi FB, Matos JL, Rizzato AP, Medeiros AH, Falco MC, Silva-Filho MC, Moura DS. 2010. SacRALF1, a peptide signal from the grass sugarcane (Saccharum spp.), is potentially involved in the regulation of tissue expansion. Plant Molecular Biology 73: 271-281.

Miyazaki S, Murata T, Sakurai-Ozato N, Kubo M, Demura T, Fukuda H, Hasebe M. 2009. ANXUR1 and 2, sister genes to FERONIA/SIRENE, are male factors for coordinated fertilization. Current Biology 19: 13271331.

Müller R, Bleckmann A, Simon R. 2008. The receptor kinase CORYNE of Arabidopsis transmits the stem cell-limiting signal CLAVATA3 independently of CLAVATA1. Plant Cell 20: 934-946.

Murphy E, De Smet I. 2014. Understanding the RALF family: a tale of many species. Trends in Plant Science 19: 664-671.

Murphy E, Vu LD, Van den Broeck L, Lin Z, Ramakrishna P, Van De Cotte B, Gaudinier A, Goh T, Slane D, Beeckman T et al. 2016. RALFL34 regulates formative cell divisions in Arabidopsis pericycle during lateral root initiation. Journal of Experimental Botany 67: 4863-4875.

Nakajima K, Sena G, Nawy T, Benfey PN. 2001. Intercellular movement of the putative transcription factor SHR in root patterning. Nature 413: 307-311.

Nishiyama T, Hiwatashi Y, Sakakibara K, Kato M, Hasebe M. 2000. Tagged mutagenesis and gene-trap in the moss, Physcomitrella patens by shuttle mutagenesis. DNA Research 7: 9-17.

Pearce G, Moura DS, Stratmann J, Ryan CA. 2001. RALF, a 5-kDa ubiquitous polypeptide in plants, arrests root growth and development. Proceedings of the National Academy of Sciences, USA 98: 12843-12847.

Plavskin Y, Nagashima A, Perroud PF, Hasebe M, Quatrano RS, Atwal GS, Timmermans M. 2016. Ancient trans-acting siRNAs confer robustness and sensitivity onto the auxin response. Developmental Cell 36: 276-289.

Qi J, Greb T. 2017. Cell polarity in plants: the Yin and Yang of cellular functions. Current Opinion in Plant Biology 35: 105-110.

Rensing SA, Goffinet B, Meyberg R, Wu SZ, Bezanilla M. 2020. The moss Physcomitrium (Physcomitrella) patens: a model organism for non-seed plants. Plant Cell 32: 1361-1376.

Rojo E, Sharma VK, Kovaleva V, Raikhel NV, Fletcher JC. 2002. CLV3 is localized to the extracellular space, where it activates the Arabidopsis CLAVATA stem cell signaling pathway. Plant Cell 14: 969-977.

Sakakibara K, Nishiyama T, Deguchi H, Hasebe M. 2008. Class 1 KNOX genes are not involved in shoot development in the moss Physcomitrella patens but do function in sporophyte development. Evolution \& Development 10: 555-566.

Schoof H, Lenhard M, Haecker A, Mayer KFX, Jürgens G, Laux T. 2000. The stem cell population of Arabidopsis shoot meristems is maintained by a regulatory loop between the CLAVATA and WUSCHEL genes. Cell 100: 635-644.
Sharma A, Hussain A, Mun BG, Imran QM, Falak N, Lee SU, Kim J, Hong J, Loake G, Ali A et al. 2016. Comprehensive analysis of plant rapid alkalization factor (RALF) genes. Plant Physiology and Biochemistry 106: 82-90.

Siezen RJ, Leunissen JAM. 1997. Subtilases: the superfamily of subtilisin-like serine proteases. Protein Science 6: 501-523.

Srivastava R, Liu JX, Guo H, Yin Y, Howell SH. 2009. Regulation and processing of a plant peptide hormone, AtRALF23, in Arabidopsis. The Plant Journal 59: 930-939.

Stegmann M, Monaghan J, Smakowska-Luzan E, Rovenich H, Lehner A, Holton N, Belkhadir Y, Zipfel C. 2017. The receptor kinase FER is a RALFregulated scaffold controlling plant immune signaling. Science 355: 287-289.

Sugano SS, Shimada T, Imai Y, Okawa K, Tamai A, Mori M, Hara-Nishimura I. 2010. Stomagen positively regulates stomatal density in Arabidopsis. Nature 463: 241-244.

Tavormina P, De Coninck B, Nikonorova N, De Smet I, Cammuea BPA. 2015. The plant peptidome: an expanding repertoire of structural features and biological functions. Plant Cell 27: 2095-2118.

Thynne E, Saur IML, Simbaqueba J, Ogilvie HA, Gonzalez-Cendales Y, Mead O, Taranto A, Catanzariti A, McDonald M, Schwessinger B et al. 2017. Fungal phytopathogens encode functional homologues of plant rapid alkalinization factor (RALF) peptides. Molecular Plant Pathology 18: 811-824.

Trifinopoulos J, Nguyen LT, von Haeseler A, Minh BQ. 2016. W-IQ-TREE: a fast online phylogenetic tool for maximum likelihood analysis. Nucleic Acids Research 44: W232-W235.

Vidali L, Bezanilla M. 2012. Physcomitrella patens: a model for tip cell growth and differentiation. Current Opinion in Plant Biology 15: 625-631.

Vidali L, Burkart GM, Augustine RC, Kerdavid E, Tüzel E, Bezanilla M. 2010. Myosin XI is essential for tip growth in Physcomitrella patens. Plant Cell 22: $1868-1882$.

Wang S, Zhou Z, Rahiman R, Lee GSY, Yeo YK, Yang X, Lau O. 2021. Light regulates stomatal development by modulating paracrine signaling from inner tissues. Nature Communications 12: 3403.

Whitewoods CD, Cammarata J, Nemec Venza Z, Sang S, Crook AD, Aoyama T, Wang X, Waller M, Kamisugi Y, Cuming A et al. 2018. CLAVATA was a genetic novelty for the morphological innovation of $3 \mathrm{D}$ growth in land plants. Current Biology 28: 2365-2376.

Wieghaus A, Prüfer D, Gronover CS. 2019. Loss of function mutation of the Rapid Alkalinization Factor (RALF1)-like peptide in the dandelion Taraxacum koksaghyz entails a high-biomass taproot phenotype. PLoS ONE 14: e0217454.

Wu J, Kurten EL, Monshausen G, Hummel GM, Gilroy S, Baldwin IT. 2007. NaRALF, a peptide signal essential for the regulation of root hair tip apoplastic $\mathrm{pH}$ in Nicotiana attenuata, is required for root hair development and plant growth in native soils. The Plant Journal 52: 877-890.

Wu SZ, Ritchie JA, Pan AH, Quatrano RS, Bezanilla M. 2011. Myosin VIII regulates protonemal patterning and developmental timing in the moss Physcomitrella patens. Molecular Plant 4: 909-921.

Xiao Y, Stegmann M, Han Z, DeFalco TA, Parys K, Xu L, Belkhadir Y, Zipfel C, Chai J. 2019. Mechanisms of RALF peptide perception by a heterotypic receptor complex. Nature 572: 270-274.

Xie Y, Zheng Y, Li H, Luo X, He Z, Cao S, Shi Y, Zhao Q, Xue Y, Zuo Z et al. 2016. GPS-Lipid: a robust tool for the prediction of multiple lipid modification sites. Scientific Reports 6: 28249.

Xu Y, Yu Z, Zhang D, Huang J, Wu C, Yang G, Yan K, Zhang S, Zheng C. 2018. CYSTM, a novel non-secreted cysteine-rich peptide family, involved in environmental stresses in Arabidopsis thaliana. Plant and Cell Physiology 59: 423-438.

Yamada M, Goshima G. 2018. KCH kinesin drives nuclear transport and cytoskeletal coalescence for tip cell growth. Plant Cell 30: 1496-1510.

Yang K, Wang L, Le J, Dong J. 2020. Cell polarity: regulators and mechanisms in plants. Journal of Integrative Plant Biology 62: 132-147.

Yi P, Goshima G. 2020. Rho of plants GTPases and cytoskeletal elements control nuclear positioning and asymmetric cell division during Physcomitrella patens branching. Current Biology 30: 1-9.

Zhao C, Zayed O, Yu Z, Jiang W, Zhu P, Hsu CC, Zhang L, Andy Tao W, Lozano-Durán R, Zhu J. 2018. Leucine-rich repeat extensin proteins regulate plant salt tolerance in Arabidopsis. Proceedings of the National Academy of Sciences, USA 115: 13123-13128. 
Zhu S, Estévez JM, Liao H, Zhu Y, Yang T, Li C, Wang Y, Li L, Liu X, Pacheco JM et al. 2020a. The RALF1-FERONIA complex phosphorylates eIF4E1 to promote protein synthesis and polar root hair growth. Molecular Plant 13: 698-716.

Zhu S, Martínez Pacheco J, Estevez JM, Yu F. 2020b. Autocrine regulation of root hair size by the RALF-FERONIA-RSL4 signaling pathway. New Phytologist 227: 45-49.

\section{Supporting Information}

Additional Supporting Information may be found online in the Supporting Information section at the end of the article.

Fig. S1 Phylogenetic incongruence between PpRALFs and AtRALFs.

Fig. S2 Ppralf mutants have no defects in branching pattern.

Fig. S3 Gametophore numbers were reduced in Ppralf mutants.

Fig. S4 Cell cycle progression was not affected in Ppralf mutants.

Fig. S5 Screening of iOX:RALF1-Citrine and iOX:RALF2Citrine overexpressors.

Fig. S6 Subcellular localisation of PpRALF1-Citrine and PpRALF2-Citrine.

Fig. S7 Screening of iOX:RALF $1^{\text {R73A }}$-Citrine overexpressor.

Fig. S8 Putative $S$-palmitoylation sites and transmembrane domain in PpRALF1 and PpRALF2.

Methods S1 Homologous recombination strategy to generate Ppralf1 knock-out.
Methods S2 Homologous recombination strategy to generate Ppralf2 knock-out.

Methods S3 Homologous recombination strategy to generate Ppralf3 knock-out.

Methods S4 Homologous recombination strategy to generate PpRALF1-Citrine knock-in.

Methods S5 Homologous recombination strategy to generate PpRALF2-tagRFP knock-in.

Methods S6 Semiquantitative RT-PCR of PpRALF1, PpRALF2 and PpRALF3 in Ppralf1,2 dko.

Table S1 Summary of BLAST queries.

Table S2 Summary of oligonucleotides.

Table S3 Summary of plasmids generated.

Table S4 Summary of transgenic lines generated.

Table S5 Summary of enriched GO terms in iOX:RALF1Citrine.

Table S6 Summary of enriched GO terms in iOX:RALF2Citrine.

Please note: Wiley Blackwell are not responsible for the content or functionality of any Supporting Information supplied by the authors. Any queries (other than missing material) should be directed to the New Phytologist Central Office. 